\title{
Magnetohydrodynamically generated velocities in confined plasma
}

\author{
Jorge A. Morales \\ LMFA, CNRS, École Centrale de Lyon, France \\ E-mail: jorge.morales-mena@ec-lyon.fr
}

Wouter J.T. Bos

LMFA, CNRS, École Centrale de Lyon, France

Kai Schneider

M2P2, CMI, CNRS, Aix-Marseille Université, France

\section{David C. Montgomery}

Department of Physics and Astronomy, Dartmouth College, NH, USA

\begin{abstract}
It is shown numerically that both toroidal and poloidal rotational flows are intrinsic to a rigid toroid confining a conducting magnetofluid in which a current is driven by the application of externally-supported electric and magnetic fields. The computation involves no microscopic instabilities or kinetic theory and is purely magnetohydrodynamic (MHD) in nature. The properties and intensity of the rotations are regulated by dimensionless numbers (Lundquist, viscous Lundquist, and Hartmann) that contain the resistivity and viscosity of the magnetofluid. The computation makes use of the recently developed "penalization method" to enforce visco-resistive boundary conditions. The point is not that other more exotic kinetic effects may not also contribute to rotational effects in toroidal laboratory devices, but rather that at the most simple magnetohydrodynamic level (uniform mass density and incompressible magnetofluids), they are not necessary. Rotational flows are an irreducible effect inherent in toroidal, driven MHD by itself, without necessary kinetic complications.
\end{abstract}




\section{Introduction}

Toroidal magnetic plasma confinement has been under investigation since the 1940s when it was recognized as a promising geometry for controlled thermonuclear fusion. Despite all the attention devoted to the idea, there are aspects of it that must be regarded as incomplete, even in theory. The difficulties in many cases reduce to the fact that there is no mathematical description of a magnetically active, dissipative plasma that is tractable, by use of even the fastest supercomputers. Time dependent electromagnetic fields combined with the particle kinetics of plasmas having the range of mass ratios represented among the various charges is simply a too large system to be susceptible to a complete treatment. Enormous simplifying assumptions have to be made to achieve any analytical/numerical progress. A common assumption has been that of an unstable ideal equilibrium whose numerous linear instabilities may reveal insight into the nonlinear dynamical behavior that is observed. It must be conceded that any description that is manageable at a detailed level will omit certain important features of a real plasma and at this stage it is to some extent a matter of taste as to which incomplete theoretical description is adopted for study.

In the following pages, we report the investigation of one such description: a voltage driven, dissipative magnetohydrodynamic (MHD) fluid with non-ideal toroidal boundaries. We omit some features that would be desirable and which seem reasonable to inject, at a later date, one at a time, into the numerical recipe we use. The principal unrealistic assumptions we make are those of uniform mass density and incompressibility, a scalar valued Newtonian viscosity, a scalar valued electrical conductivity, and the omission of a finite thermal conductivity (it will be seen that in effect an infinite thermal conductivity has been assumed, since no thermal effects are allowed to develop except those associated with the incompressible velocity field). Despite what appear to be these gross oversimplifications, what remains is at the very perimeter of what is computable if we intend to stay with arbitrary initial configurations which are not in equilibrium, and to follow through with enforcing viscous and resistive boundary conditions.

What is of particular interest is the spontaneous development of both toroidal and poloidal rotation of the bulk magnetofluid as a whole. It is not physically obvious that this should happen, even though it has been known for some time to occur in toroidal laboratory devices [1]. The importance of non-zero velocities in the MHD description of toroidally confined plasma was realized by Pfirsch and Schlüter [2], however without taking into account all the different terms in the force balance. We will take into account all these terms. The resulting flow pattern is presented here as a computational fact, still in need of a clear or simple physical explanation. The degree of the two types of rotation are seen to depend upon several things, such as the Reynolds-like dimensionless numbers assumed for the magnetofluid; the geometry of the toroid, which is allowed to have variable cross sections; the safety factor of the magnetofluid; and perhaps others.

The numerical technique employed is relatively recent, and descends from what has been called the volume penalization technique [3], originally developed for 
hydrodynamics. The entire computational domain is assumed to be three-dimensional and spatially periodic, so that pseudospectral methods can be employed, taking advantage of the fast Fourier transform and eliminating some complications associated with imposing incompressibility of the velocity and magnetic fields. Then a toroidal volume is carved out within the volume of one period in each direction. Inside the toroid, the transport coefficients of viscosity and resistivity are assumed small but nonzero. Outside the toroid mechanical and magnetic activity are suppressed using the penalization technique [4]. A steep gradient between the two regions serves as an active visco-resistive boundary. The method has been used to considerable effect both for Navier-Stokes turbulence [5] and for magnetofluids in the recent past [6, 7, 4]. The Fourier pseudo-spectral codes used for the dynamical advancement of the field quantities are of a well-studied type.

In Sec. 2, we fix the geometry of the confined magnetofluid and write down the system of equations and boundary conditions that will govern the dynamics. An external forcing of the magnetic field provides the toroidal electric field which initiates and drives the current. A vacuum toroidal dc magnetic field, regarded as externally supported from outside the system, is also assumed to be present. In addition another toroidal component of the magnetic field is allowed to develop in time if the dynamics so dictate.

In Sec. 3, the results are presented. They are divided in four different parts. The first discusses the generation of toroidal velocities for a dissipative system. The second exposes the results where the nonlinear term is dominant and a comparison is made between different toroidal geometries. In the third section we study the effect of the variation of the safety factor and in the last part how the system evolves if the imposed toroidal magnetic field is inverted. We illustrate in detail the development of the driven magnetofluid configurations and the development of spontaneous toroidal rotation.

\section{Geometrical configuration and governing equations}

In the MHD approximation the plasma is described as a charge-neutral conducting fluid. Despite its low complexity compared to kinetic descriptions it can give rise to a wealth of intricate phenomena and its analytical treatment is only possible in some simplified cases, either in the absence of velocity fields [8,9] or in the absence of non-linear interactions [10]. If one considers the complete problem one necessarily needs to consider a discretized numerical approximation of the full nonlinear system. The equations we consider are the dimensionless incompressible viscoresistive MHD equations for the velocity field $\boldsymbol{u}$ and for the magnetic field $\boldsymbol{B}$, in 'Alfvénic' units [11].

$$
\begin{aligned}
& \frac{\partial \boldsymbol{u}}{\partial t}-M^{-1} \nabla^{2} \boldsymbol{u}=-\nabla\left(P+\frac{1}{2} \boldsymbol{u}^{2}\right)+\boldsymbol{u} \times \boldsymbol{\omega}+\boldsymbol{j} \times \boldsymbol{B}, \\
& \frac{\partial \boldsymbol{B}}{\partial t}=-\nabla \times \boldsymbol{E}, \\
& \boldsymbol{E}=S^{-1} \boldsymbol{j}-\boldsymbol{u} \times \boldsymbol{B}, \\
& \nabla \cdot \boldsymbol{u}=0, \quad \nabla \cdot \boldsymbol{B}=0,
\end{aligned}
$$


with the current density $\boldsymbol{j}=\nabla \times \boldsymbol{B}$, the vorticity $\boldsymbol{\omega}=\nabla \times \boldsymbol{u}$, the pressure $P$ and the electric field $\boldsymbol{E}$. These equations are non-dimensionalized using the toroidal Alfvén speed $C_{A}=B_{\text {ref }} / \sqrt{\rho \mu_{0}}$ as typical velocity, with $B_{\text {ref }}=1.2$ the reference toroidal magnetic field at the center of the torus $\left(R=R_{0}=0.55 \pi \approx 1.73\right.$ for both considered geometries), $\rho$ the density and $\mu_{0}$ the magnetic constant. We will exclusively consider two toroidal geometries with differently shaped cross-sections (see Fig. 1). The reference length $L$ is the diameter of the cross section for the circular case and is the minor diameter for the asymmetric ' $\mathrm{D}$ ' shape ( $L=0.6 \pi \approx 1.88$ for both geometries). The ' $\mathrm{D}$ ' shape parametric equation is a modified version of the formula given by Manickam [12],

$$
\begin{aligned}
& R(t)=\frac{L}{2}[\cos (t-\alpha+\delta \sin (t)) \cos (\zeta)-\kappa \sin (t) \sin (\zeta)], \\
& Z(t)=\frac{L}{2}[\cos (t-\alpha+\delta \sin (t)) \sin (\zeta)+\kappa \sin (t) \cos (\zeta)],
\end{aligned}
$$

with $t \in[0,2 \pi], \delta$ the triangularity, $\kappa$ the ellipticity, $\alpha$ the asymmetry and $\zeta$ the rotation angle. For the considered asymmetric cross section the following values of these parameters are chosen: $\delta=0.5, \kappa=2.1, \alpha=0.4$ and $\zeta=0.15$.

The MHD equations are completed by the initial and boundary conditions of the problem, and two dimensionless quantities: the viscous Lundquist number $(M)$ and the Lundquist number $(S)$ defined as

$$
M=\frac{C_{A} L}{\nu}, \quad S=\frac{C_{A} L}{\lambda},
$$

with $\lambda$ the magnetic diffusivity and $\nu$ the kinematic viscosity. The ratio of these two quantities is the magnetic Prandtl number $\operatorname{Pr}=\nu / \lambda$, which we have chosen unity in the present study, thereby reducing the number of free parameters, which characterize the magnetofluid, to one, the viscous Lundquist number, $M$. Previous investigations indicate that it is the geometric mean of the viscosity and the magnetic diffusivity which is important to the dynamics $[13,14]$. In setting the Prandtl number to one, a change in the viscous Lundquist numbers, $M$ or $S$, is equivalent to a change in the Hartmann number.

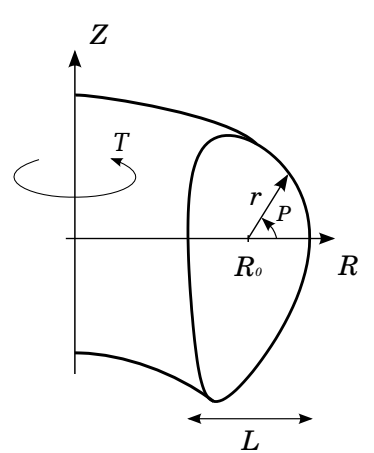

Asymmetric ' $\mathrm{D}$ '

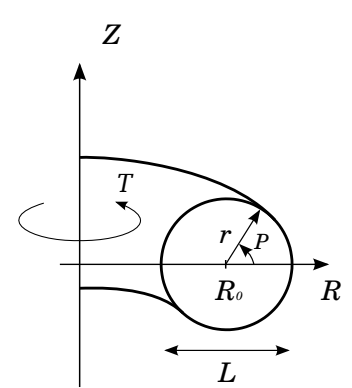

Circular

Figure 1. Cross-sections of the toroidal geometries considered in the present work. The toroidal direction is labelled $T$ and the poloidal $P$. 
In the ideal MHD framework a scalar-pressure equilibrium state is assumed in which $\boldsymbol{u}=0$,

$$
\boldsymbol{j} \times \boldsymbol{B}=\nabla P
$$

This equilibrium is possible in a cylindrical geometry, for instance in $z$ - and $\theta$-pinches. It is shown in $[15,16]$ that in the case of finite conductivity such an equilibrium is not possible in a toroidal geometry if irrotational toroidal magnetic and electric fields are applied. A steady state in Faraday's law imposes the toroidal electric field to be irrotational in the region of interest. The chosen spatial dependence for $\boldsymbol{E}_{0_{T}}$ is $\propto 1 / R$. In the simple case of a space-uniform conductivity, which we consider in the present study, the current density has the same dependence. The form for the imposed toroidal magnetic field, which is also proportional to $1 / R$, comes from the integration of Ampère's law on a toroidal loop. So the externally imposed magnetic field and toroidal, laminar, voltage-driven current density are given by,

$$
\boldsymbol{B}_{0_{T}}(R) \propto \frac{R_{0}}{R} \boldsymbol{e}_{T}, \quad \boldsymbol{J}_{0_{T}}(R) \propto \frac{R_{0}}{R} \boldsymbol{e}_{T} .
$$

The toroidal magnetic and current density profiles give the imposed threedimensional helical magnetic field $\boldsymbol{B}_{0}=\boldsymbol{B}_{0_{T}}+\boldsymbol{B}_{0_{p o l}}$, with $\boldsymbol{B}_{0_{p o l}}=B_{0_{R}} \boldsymbol{e}_{R}+B_{0_{Z}} \boldsymbol{e}_{Z}$. The poloidal magnetic field is calculated from the current density distribution $\boldsymbol{J}_{0_{T}}(R)$. For the details of generating the poloidal magnetic field in general geometries numerically we refer to Appendix A. Here $\boldsymbol{e}_{T}, \boldsymbol{e}_{R}$ and $\boldsymbol{e}_{Z}$ are unit vectors in the toroidal/azimuthal, radial and vertical directions respectively (Fig. 1).

The toroidal magnetic field magnitude is tuned to have an edge safety factor $q=$ $\overline{\left.r B_{0_{T}}\right|_{\text {wall }}} / R_{0} \overline{\left.B_{0_{P}}\right|_{\text {wall }}}=5.7$ for the asymmetric geometry and $q=3.3$ for the symmetric cross section. A bar over a symbol indicates an average over the entire boundary. These safety factor values will be used for the majority of studied cases. The pinch-ratio associated to these values of $q$, defined as the ratio between the wall-averaged poloidal and the volume-averaged toroidal imposed magnetic field, $\Theta=\overline{B_{0_{P}}} /\left\langle B_{0_{T}}\right\rangle=0.16$, is the same for both geometries. The resulting three-dimensional magnetic field lines are visualized for both geometries in Fig. 2.
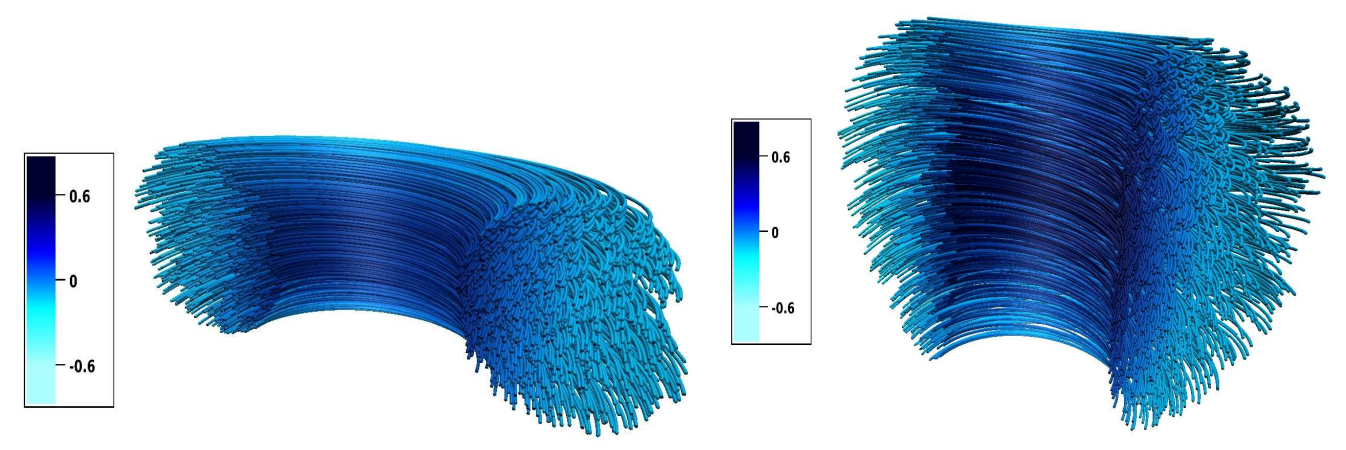

Figure 2. Three-dimensional magnetic field lines colored with the vertical magnetic field $\left(B_{Z}\right)$. For the symmetric (left) and asymmetric (right) cross sections. 
The Lorentz force resulting from the calculated poloidal field $\boldsymbol{B}_{0_{p o l}}$ and the imposed toroidal current density $\boldsymbol{J}_{0_{T}}$ is not curl-free $[15,16]$. Since the curl of a pressure gradient is necessarily zero, the equilibrium described by (8) becomes impossible and additional terms of Eq. (1) need to be taken into account to balance the equation. Since all other terms in (1) are proportional to (or quadratic in) the velocity, the resulting state must be dynamic. In other words if we take the curl of Eq. (1) we end with the vorticity equation,

$$
\frac{\partial \boldsymbol{\omega}}{\partial t}-M^{-1} \nabla^{2} \boldsymbol{\omega}-\nabla \times(\boldsymbol{u} \times \boldsymbol{\omega})=\nabla \times(\boldsymbol{j} \times \boldsymbol{B}) \neq 0,
$$

we observe that if the Lorentz force term is not curl-free, it acts as a source of vorticity: a toroidal plasma, described by viscoresistive MHD, confined by curl-free toroidal electric and magnetic fields, necessarily moves.

It is true that the rationale described above depends on the choice of the electric conductivity, which was assumed to be uniform. It was however shown [17] that to satisfy (8) in a torus, very unusual profiles of the electrical conductivity must be assumed. The simple case of constant magnetic resistivity is then treated in this study. The case of non-uniform resistivity profiles is one of our most important perspectives.

It follows from the foregoing that it is necessary to take into account all other terms in the MHD equations, and analytical treatment becomes impossible unless symmetries are assumed. To study the full dynamics we are obliged to solve numerically the system and this is what is done in the present investigation. Equations (1)-(4) are discretized with a Fourier pseudo-spectral method on a Cartesian grid. To impose the boundary conditions we use the volume-penalization technique, a method of the immersed boundary type. Results for two-dimensional viscoresistive MHD can be found in Ref. [6, 4]. The method is presented in detail for three-dimensional viscoresistive MHD equations in [7]. The study exposed in the present paper is the numerical study of confined MHD using the toroidal geometries shown in Fig. 1.

The total magnetic field is decomposed into a base component and a perturbation,

$$
\boldsymbol{B}=\boldsymbol{B}_{0}+\boldsymbol{B}^{\prime}
$$

Numerically only the perturbation of the magnetic field is computed, the base magnetic field, $\boldsymbol{B}_{0}$, computed from (9) is fixed and it is introduced in the Navier-Stokes equation and in the induction equation as follows,

$$
\begin{aligned}
& \frac{\partial \boldsymbol{u}}{\partial t}-M^{-1} \nabla^{2} \boldsymbol{u}=-\nabla\left(P+\frac{1}{2} \boldsymbol{u}^{2}\right)+\boldsymbol{u} \times \boldsymbol{\omega}+\left(\boldsymbol{j}^{\prime}+\boldsymbol{j}_{0}\right) \times\left(\boldsymbol{B}^{\prime}+\boldsymbol{B}_{0}\right)(12 \\
& \frac{\partial \boldsymbol{B}^{\prime}}{\partial t}-S^{-1} \nabla^{2} \boldsymbol{B}^{\prime}=\nabla \times\left[\boldsymbol{u} \times\left(\boldsymbol{B}^{\prime}+\boldsymbol{B}_{0}\right)\right]
\end{aligned}
$$

To close the equations we have the incompressibility of the velocity field and the solenoidal constraint on the perturbed part of the magnetic field,

$$
\nabla \cdot \boldsymbol{u}=0, \quad \nabla \cdot \boldsymbol{B}=0 .
$$


The boundary conditions are to be no-slip, $\left.\boldsymbol{u}\right|_{\text {wall }}=0$, for the velocity. For the magnetic perturbation, the poloidal component and the component normal to the wall vanish,

$B^{\prime} P_{\text {wall }}=B^{\prime}{ }_{\perp_{\text {wall }}}=0$, while the toroidal component is free. The normal component $B_{\perp}$ vanishing at the wall physically corresponds to perfectly conducting boundary conditions. The zero poloidal fluctuations $B_{P_{\text {wall }}}^{\prime}$ are imposed for numerical convenience. Since the perturbed magnetic field remains small compared to the field $\boldsymbol{B}_{0}$ in the present investigation, we do not think that this simplification significantly influences the results.

The initial condition for the simulations is zero magnetic perturbations and zero velocity. The simulations are carried out on a cubic domain of size $(2 \pi)^{3}$ for the asymmetric and $(2 \pi \times 2 \pi \times \pi)$ for the symmetric cross section consisting of $256^{3}$ grid points. We fix the penalization parameter to $\eta=5 \cdot 10^{-4}$. The time step is adaptive and the chosen CFL coefficient is 0.1 .

\section{Results and discussion}

The results are divided into four different parts. The first shows the solution of the simulations at a low viscous Lundquist number, where an illustration of the generation of toroidal velocities is presented. The second exposes the calculations at higher viscous Lundquist, where the flow behavior of the plasma changes towards a dominantly toroidal flow. In the third section we compare, at fixed transport coefficients, simulations carried out for different safety factors and in the fourth section we show the results when the toroidal magnetic field is reversed.

\subsection{Generation of toroidal velocities at low viscous Lundquist number}

In this section the calculations are performed for a low viscous Lundquist number, $M=23$, in the geometry with symmetric cross section and $q=3.3$. All the results are presented when the system has reached a statistically stationary state.

Fig. 3 shows the presence of a poloidal flow, a pair of counterrotating vortices in the poloidal plane. In this case the flow topology is almost axisymmetric with respect to the $Z$-axis. To visualize more clearly the toroidal velocities and the double poloidal recirculation, the azimuthally averaged velocity field is presented in Fig. 4. We distinguish four different zones, where the toroidal velocity changes sign, and the already mentioned "double smoke ring". Indeed, in the limit of vanishing nonlinearity, Bates and Montgomery [10] showed analytically that the steady state solution is a pair of poloidally rotating vortices, aligned with the toroidal direction.

The origin of toroidal velocities was demonstrated for vanishing viscous Lundquist in a rectangular cross section [18]. For a circular cross section and at low $M$ number we will illustrate the generation of this velocity component. First, we illustrate that the forcing appearing in the vorticity equation (10) creates a toroidal vorticity with opposite sign in relation to the mid-plane of the torus (see Fig. $5(a)$ ). This creates automatically a radial velocity that will interact with the imposed toroidal magnetic 


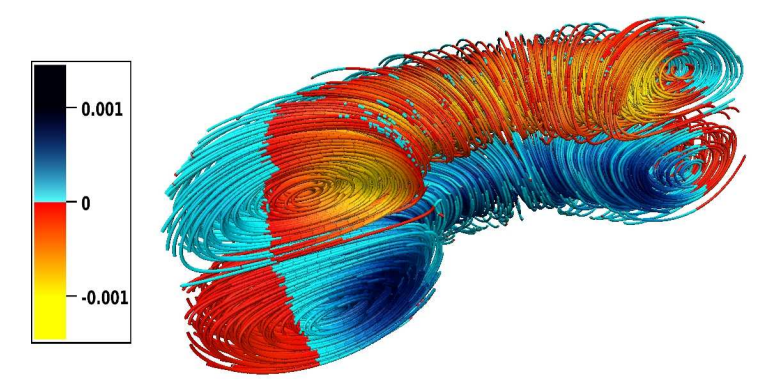

Figure 3. Streamlines colored with toroidal velocity $\left(u_{T}\right)$ for $M=23$.

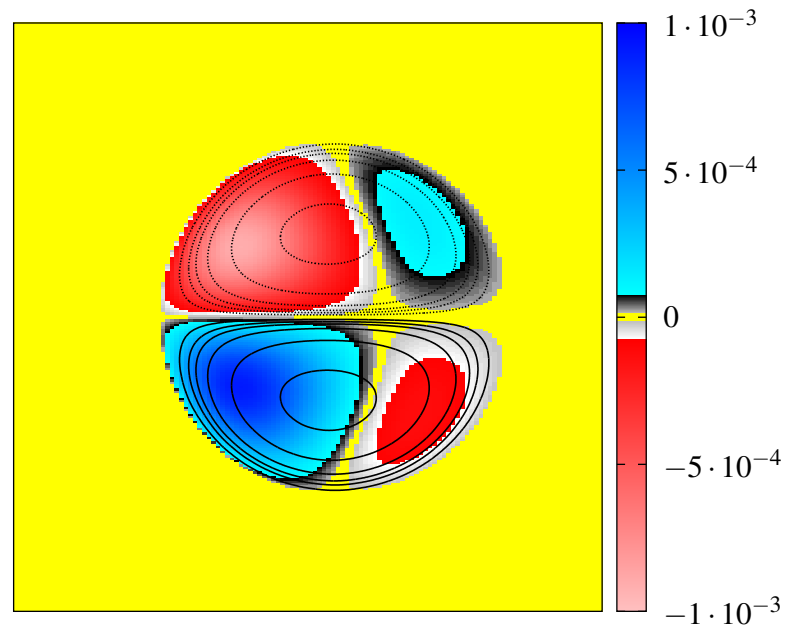

Figure 4. Azimuthally averaged toroidal velocity and poloidal stream function contours (solid line positive, dotted line negative contours).

field (Fig. $5(b)$ ). The interaction will produce a perturbation to the toroidal magnetic field $\left(B_{T}^{\prime}\right)$. Notice that this magnetic field will have positive and negative areas located in a similar position as the radial velocity (Figs. $5(b)$ and $(c)$ ). It was shown [18] that the equation giving the first order perturbed toroidal magnetic component $B_{T}^{(1)}$ is,

$$
\nabla^{2}\left(B_{T}^{\prime(1)} \boldsymbol{e}_{T}\right) \sim-u_{R} \frac{B_{T_{r e f}}}{R^{2}} \boldsymbol{e}_{T} .
$$

The sign of the right hand side, will only depend on the sign of $u_{R}$ and of the imposed toroidal field $B_{T_{\text {ref }}}$.

It follows that the curl of the perturbed toroidal magnetic field $\left(B_{T}^{\prime}\right)$ will produce a poloidal current density, $\boldsymbol{j}_{\text {pol }}=\nabla \times B_{T}^{\prime}$ (Fig. $6(a)$ ). The imposed poloidal magnetic field $\boldsymbol{B}_{0_{p o l}}$ will then interact with the perturbed current density $\boldsymbol{j}_{\text {pol }}$ to create a toroidal Lorentz force (see Figs. $6(b)$ and $(c)$ ). The Lorentz force will finally induce toroidal 
velocities. Note that there is a similarity in the negative and positive zones between the toroidal velocity and the toroidal Lorentz force fields (see Figs. 4 and $6(c)$ ). We note that the sign in the toroidal Lorentz force depends exclusively on the angle between $\boldsymbol{j}_{\text {pol }}$ and $\boldsymbol{B}_{0_{p o l}}$. As a consequence this angle influences directly the toroidal velocity direction.

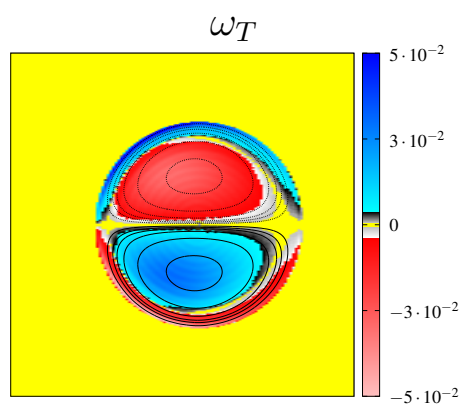

(a)

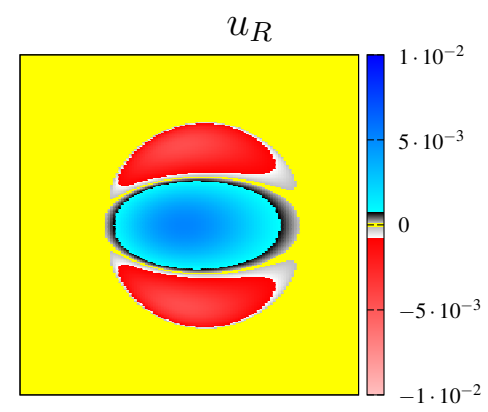

(b)

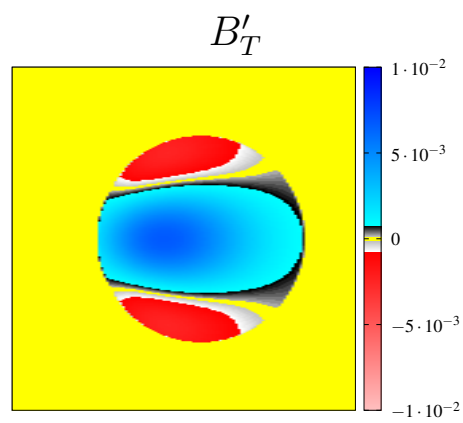

(c)

Figure 5. Azimuthally averaged: (a) Toroidal vorticity $\omega_{T}$ and poloidal stream function, (b) radial velocity $u_{R}$ and $(c)$ perturbation of the toroidal magnetic field, $B_{T}^{\prime}$.

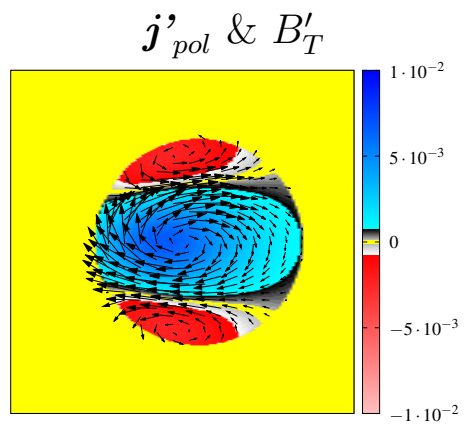

(a)

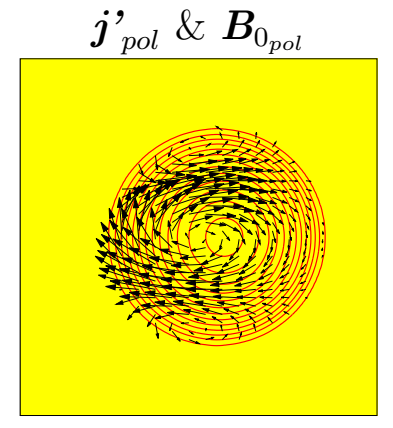

(b)

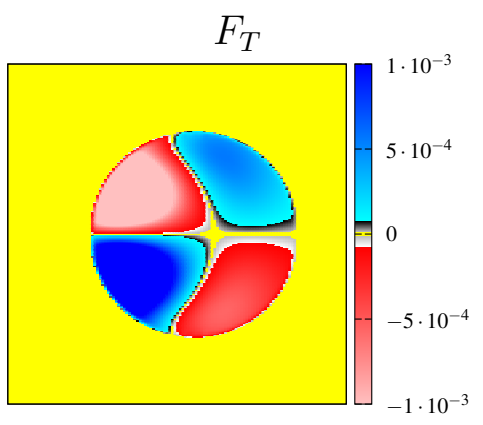

$(c)$

Figure 6. Azimuthally averaged: (a) Poloidal current density $\boldsymbol{j}_{\text {pol }}$ (vectors) and perturbation of the toroidal magnetic field $B_{T}^{\prime},(b)$ current density $\boldsymbol{j}_{\text {pol }}$ (vectors) and imposed poloidal magnetic field lines $\boldsymbol{B}_{0_{p o l}}$ and $(c)$ toroidal Lorentz force $F_{T}$.

Another way, to apprehend the fact that the poloidal flow interacts first with the magnetic field creating subsequently toroidal velocities, is to see the time evolution of the different velocity components. The velocities in the poloidal plane (in the poloidal direction $P$ and in the minor radius direction $r$ ) grow first. After that the toroidal velocity is generated (see Fig. 7).

At low viscous Lundquist number the dominant velocities are in the poloidal plane and form two counterrotating vortices. Small toroidal velocities appear and they form a quadrupole with alternating positive and negative directions. The analytical results published by Bates and Montgomery [10] are in good agreement. Also the numerical generation of toroidal velocities agrees with the calculations made by Kamp et al. [18]. 


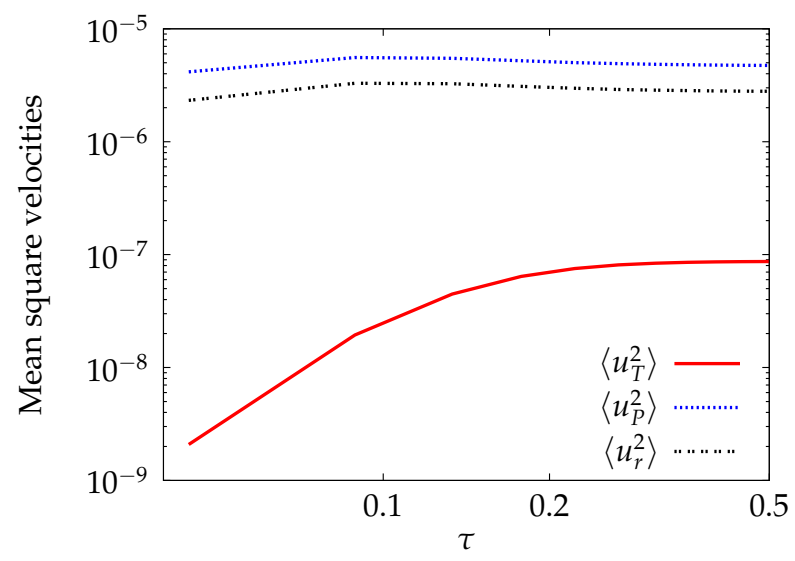

Figure 7. Poloidal and toroidal square velocity component evolutions at early times, in toroidal Alfvénic time units $(\tau)$.

\subsection{Simulations for higher viscous Lundquist numbers}

In this section the calculations are made for the asymmetric cross section with fixed $q=5.7$ and for the circular cross section, $q=3.3$. The viscous Lundquist numbers are modified changing the transport coefficients $\nu$ and $\lambda$ (with $\operatorname{Pr}=1$ ), keeping the geometry and the reference toroidal magnetic field unchanged, $B_{r e f}=1.2$.

With higher viscous Lundquist numbers it takes longer for the system to reach the saturated state. In the first instants an oscillatory behavior is present (see for example the different energy evolutions in Figs. 8 and 9). The kinetic and the fluctuating magnetic energy oscillate in opposition of phase, but these oscillations are damped out in a finite time. In the following section we will analyse and compare the different simulations when the system has reached this non-oscillatory steady state.
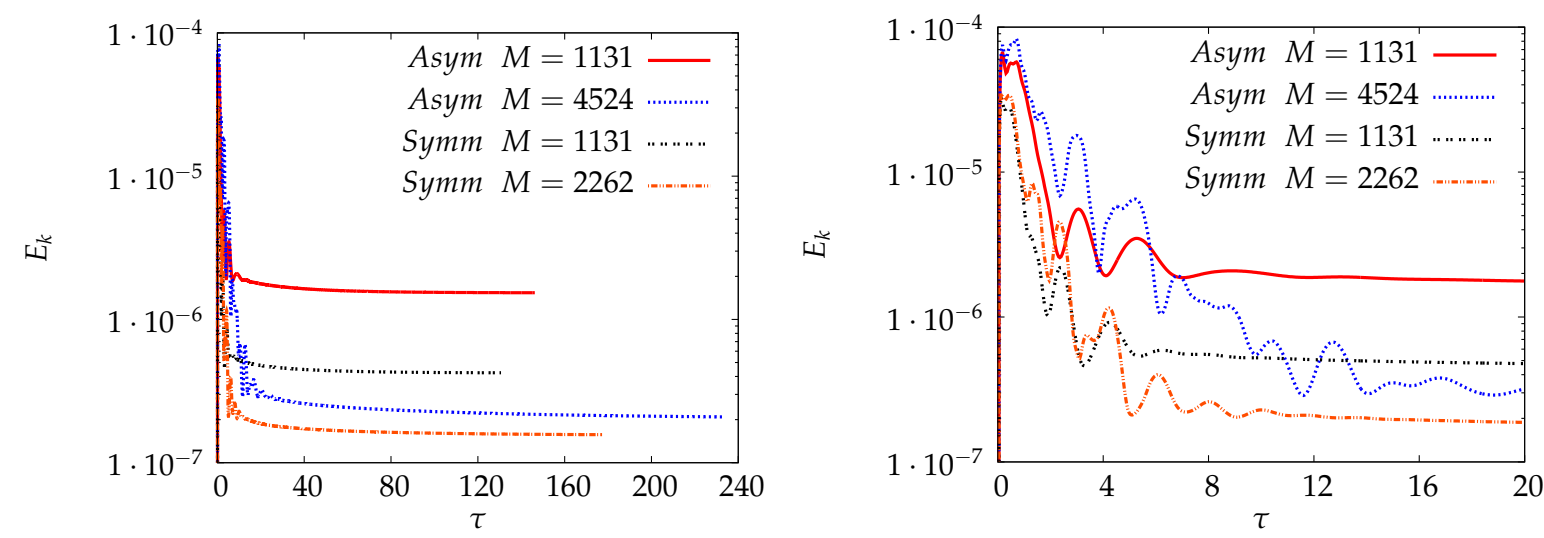

Figure 8. Kinetic energy evolution at large times (left) and oscillatory behavior at early time (right) in toroidal Alfvénic time units, for asymmetric and symmetric geometry.

The calculations with increasing viscous Lundquist number show an important change in the fluid flow. The previously small toroidal velocities increase considerably and will become more important, in magnitude, than the poloidal plane velocities. For 

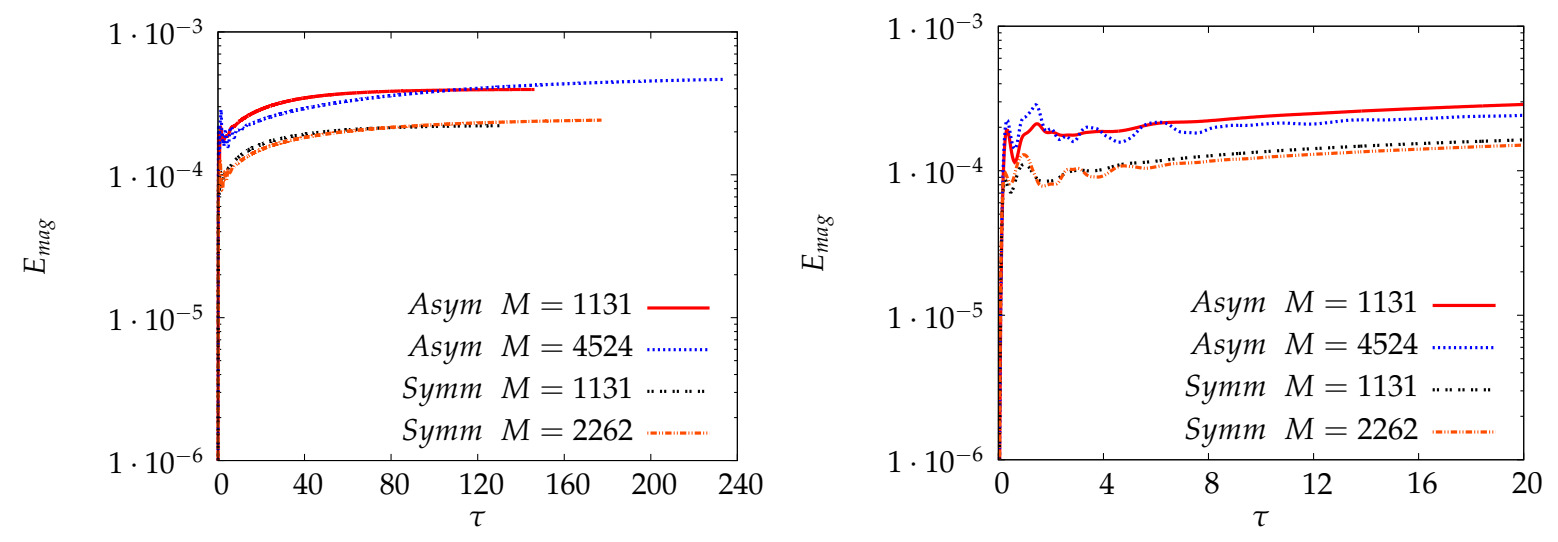

Figure 9. Fluctuating magnetic energy evolution at large times (left) and oscillatory behavior at early time (right) in toroidal Alfvénic time units, for asymmetric and symmetric geometry.

nonzero nonlinearity, i.e., by increasing $M$, the vortices start moving in the toroidal direction. The toroidal velocity increases with $M$ in the two considered geometries. The three dimensional velocity streamlines show a substantial change of topology, from dominantly poloidal to dominantly toroidal flow (see Figs. 10 and 11).
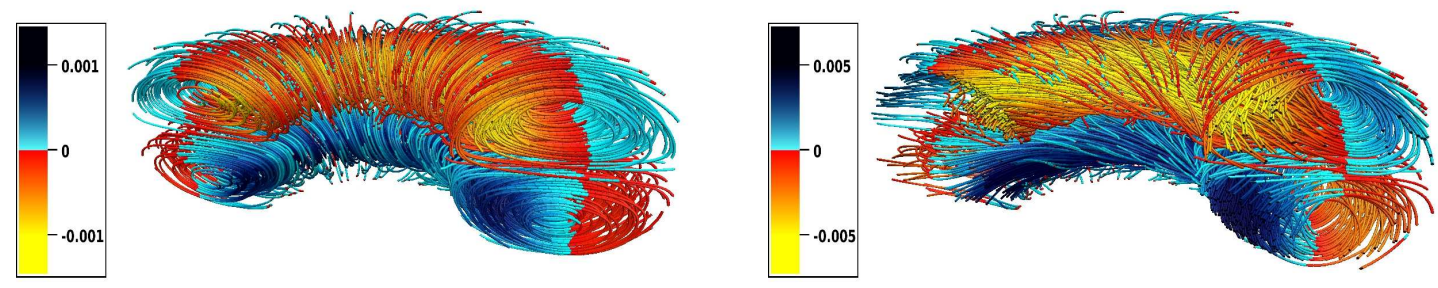

Figure 10. Streamlines colored with toroidal velocity $\left(u_{T}\right)$ for $M=23$ (left) and $M=226$ (right) for the symmetric torus.

The flow evolution is quantified in Fig. 12, where we observe that the principal direction of the flow is toroidal if $M$ is raised beyond $\sim 40$. The square toroidal velocity saturates for increasing $M$ at a value of $\sim 80 \%$ of the total square velocity for the asymmetric cross section and at $\sim 60 \%$ for the circular profile. This toroidal organization of the flow is consistent with the tendency of the velocity field to align with the magnetic field, as is illustrated in Fig. 13, where we compute the average (over the toroidal domain) of the absolute value of the cosine of the angle between the velocity and magnetic field. This quantity is equal to one if the velocity and the magnetic field are perfectly aligned or antialigned. The evolution of the ratio $\left\langle u_{T}^{2}\right\rangle /\left\langle\left|\boldsymbol{u}^{2}\right|\right\rangle$ with $M$ shows the same trend as the alignment between the magnetic and the velocity field. 

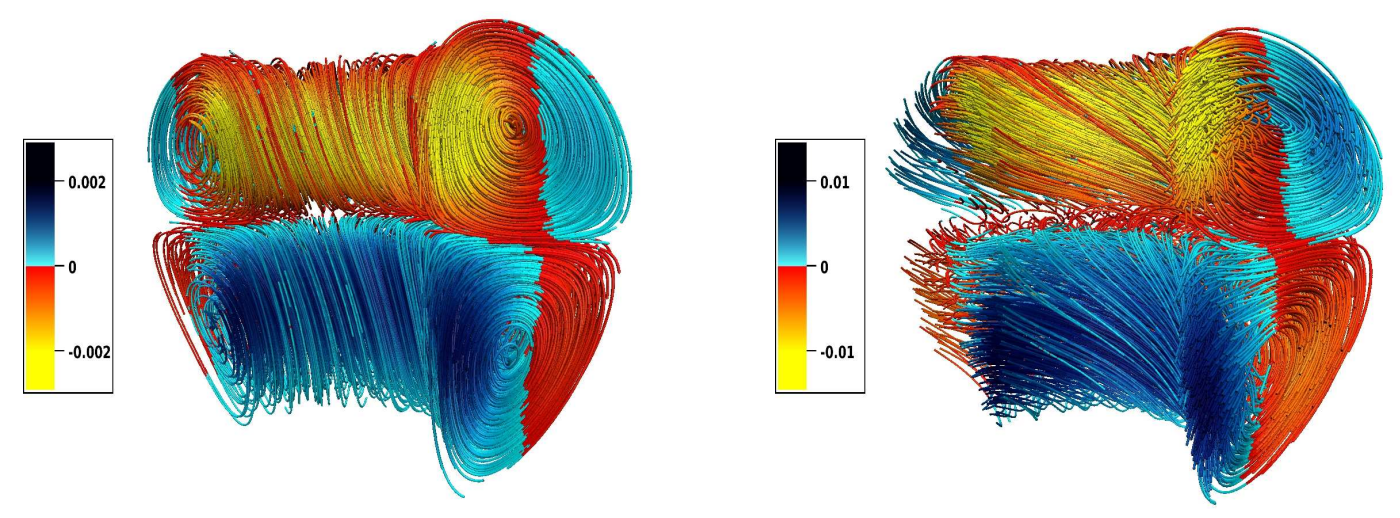

Figure 11. Streamlines colored with toroidal velocity $\left(u_{T}\right)$ for $M=23$ (left) and $M=226$ (right) for the asymmetric torus.

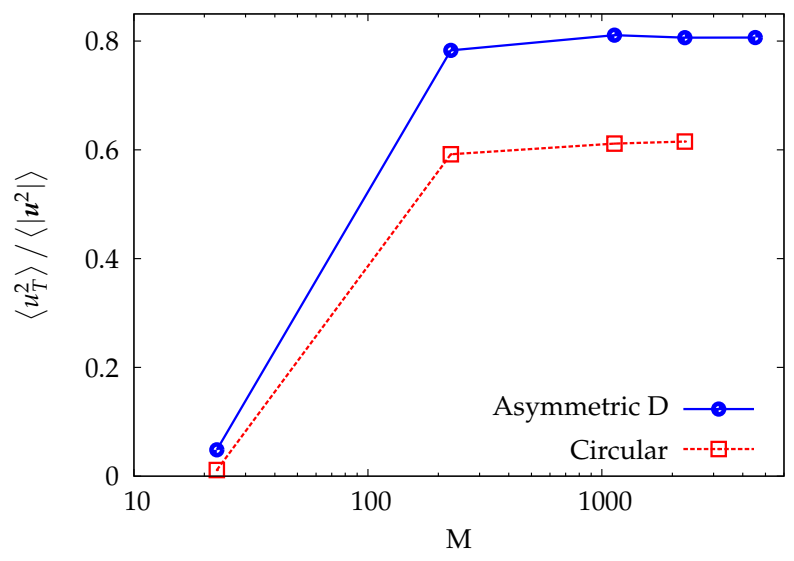

Figure 12. The ratio of the mean-square toroidal velocity to the total mean-square $\left\langle u_{T}^{2}\right\rangle /\left\langle\left|\boldsymbol{u}^{2}\right|\right\rangle$ as a function of $M$.

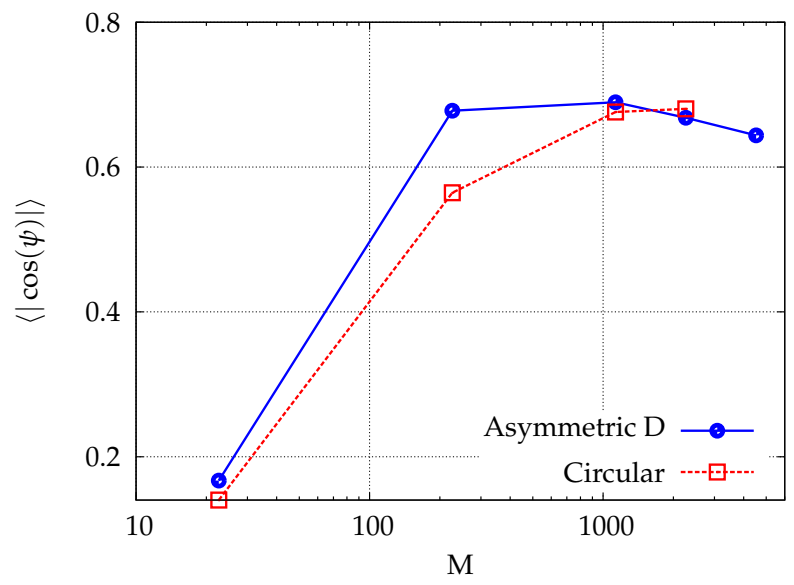

Figure 13. Average over the domain of the absolute value of the cosine of the angle between the velocity field and magnetic field.

An important difference is observed between the flows that are generated in the 
two geometries. The volume averaged toroidal angular momentum is defined by

$$
\left\langle L_{T}\right\rangle=\frac{1}{V} \int_{V} R u_{T} d V
$$

For the torus with circular cross section, this quantity is zero to a good computational approximation $\left(<10^{-15}\right)$. The up-down anti-symmetry of the velocity field is responsible for this absence of toroidal angular momentum. However, for the torus with asymmetric cross section this is not the case. There is a symmetry breaking of the flow and the volume integral of the toroidal velocity is nonzero. In our calculations this can be visualized in the azimuthally averaged velocity fields in Fig. 14. It is more clear for the last case, at $M=4524$, that the positive toroidal velocity occupies a larger part of the poloidal plane than the negative toroidal velocity. To quantify the amount of dissymmetry in the flow we present the evolution of the normalized toroidal angular momentum with $M$ (see Fig. 15). This quantity increases with the viscous Lundquist number. This up-down symmetry effect is in agreement with timeindependent computations [11] and also with gyrokinetic simulations and experiments $[19,20]$.

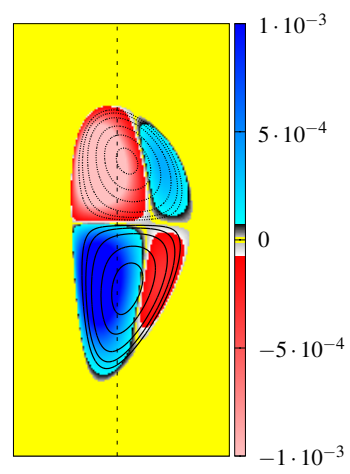

(a)

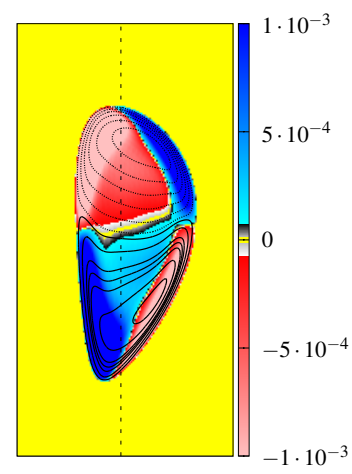

(b)

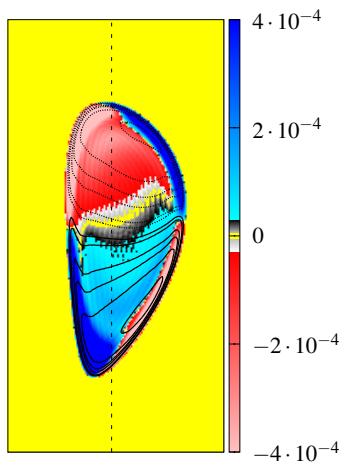

(c)

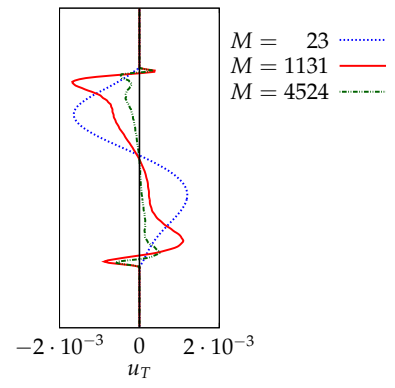

(d)

Figure 14. Azimuthally averaged flow visualizations: toroidal velocity $u_{T}$ and poloidal stream function contours (solid line positive, dotted line negative contours) for $M=23(a), M=1131(b)$ and $M=4524(c)$. (d) Toroidal velocity profiles along a vertical cut. The position of these cuts is indicated in $(a),(b)$ and $(c)$ by a dotted vertical line.

Furthermore in Fig. 14 we can observe the two counterrotating vortices. They are still present at higher viscous Lundquist but undergo a deformation and their center is shifted outwards. The larger toroidal velocities concentrate near the boundaries as well as the poloidal speeds (this can be seen from the stream function isocontours that tend to converge near the boundaries). Nevertheless the velocity magnitude is globally less important for high $M$. In fact the kinetic energy has a maximum and then decreases if the viscous Lundquist number is raised (see Fig. 16). This behavior is explained by the decrease of the magnitude of the Lorentz force with the viscous Lundquist number in the center of the domain. Indeed, the plasma seems to self-organize to a state with a forcefree region in the center, a behavior also observed for straight-cylinder computations at 
high pinch ratio [21]. The evolution with $M$ of the root mean square (RMS) value of this force is presented in Fig. 17. The spatial distribution of the norm of the Lorentz force vector in the poloidal plane is visualized for the asymmetric geometry in Fig. 18. The vanishing of the Lorentz force in the core comes from the alignment between the magnetic and current density fields. A measure giving the alignment between these three-dimensional quantities is the volume-averaged current helicity defined as

$$
H_{j}=\left\langle\frac{\boldsymbol{j} \cdot \boldsymbol{B}}{\|\boldsymbol{j}\|\|\boldsymbol{B}\|}\right\rangle \text {. }
$$

We observe (Fig. 19) that for increasing viscous Lundquist number the global current density and magnetic field tend to be oriented in the same direction, the quantity in the figure approaches the unit value. This causes the Lorentz force term to decrease for higher $M$ in the center of the domain, the magnitude of the imposed toroidal current density and magnetic fields remaining constant.

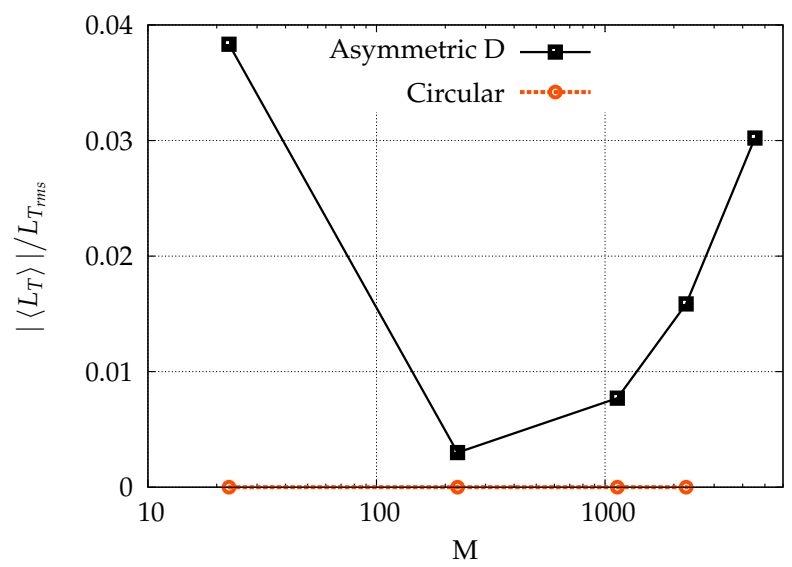

Figure 15. Normalized toroidal angular momentum $\left|\left\langle L_{T}\right\rangle\right| / L_{T_{r m s}}$ as a function of $M$ observed in the tori with asymmetric and symmetric cross section, respectively.

Mainly, the variation of the alignment between $\boldsymbol{j}$ and $\boldsymbol{B}$ occurs in the poloidal plane. To quantify the alignment among the poloidal current density and the poloidal magnetic field we compute the volume-averaged absolute value of the cosine of the angle between these two fields,

$$
\langle|\cos \Phi|\rangle=\left\langle\frac{\left|\boldsymbol{j}_{p o l} \cdot \boldsymbol{B}_{p o l}\right|}{\left\|\boldsymbol{j}_{p o l}\right\|\left\|\boldsymbol{B}_{p o l}\right\|}\right\rangle,
$$

where $\boldsymbol{J}_{p o l}$ and $\boldsymbol{B}_{p o l}$ are the projections of $\boldsymbol{J}$ and $\boldsymbol{B}$ on the poloidal plane. This quantity at low viscous Lundquist is smaller compared to the value of the current helicity at the same $M$ number (see Figs. 19 and 20). With increasing viscous Lundquist the cosine of this angle grows and approaches unity. There is a stronger change in the alignment between the current density and magnetic field in the poloidal plane. This poloidal alignment makes the toroidal Lorentz force vanish in the core of the domain.

Whether or not the Lorentz force term reaches an asymptote at higher $M$ or if a transition to another state exists remains an open question. 


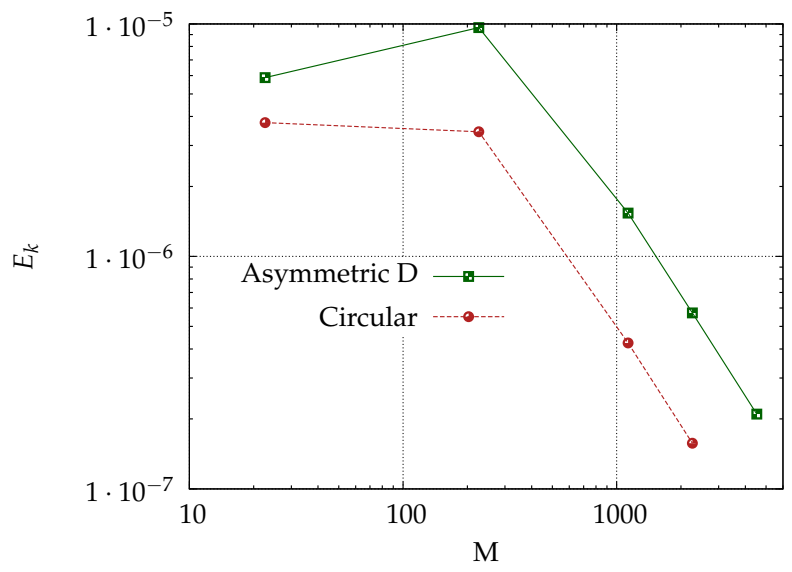

Figure 16. Kinetic energy as a function of $M$ for the asymmetric and symmetric cross sections.

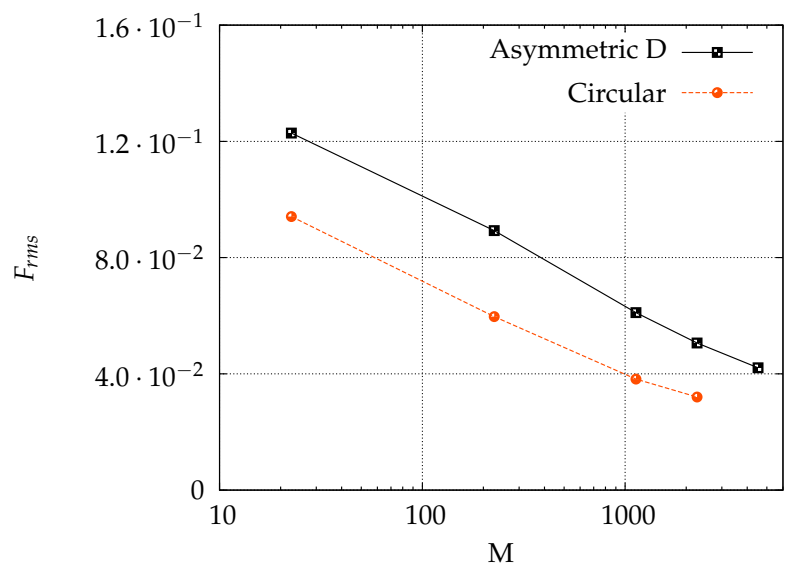

Figure 17. Root mean square value of the Lorentz force as a function of the viscous Lundquist number.

The system is almost axisymmetric around $Z$ but small fluctuations around the toroidally averaged fields exist, defined as

$$
\tilde{\boldsymbol{u}}=\boldsymbol{u}-\langle\boldsymbol{u}\rangle_{T}, \quad \tilde{\boldsymbol{B}}^{\prime}=\boldsymbol{B}^{\prime}-\left\langle\boldsymbol{B}^{\prime}\right\rangle_{T} .
$$

The most important normalized fluctuations around the axisymmetric state are in the velocity field (Fig. 21), they are localized at the boundaries (see Fig. 23). The evolution of the normalized kinetic and magnetic fluctuations as a function of the viscous Lundquist number are presented respectively in Figs. 21 and 22. For the highest viscous Lundquist, $M=4524$ and asymmetric cross section, we have the maximum ratio $\tilde{\boldsymbol{u}}_{r m s} / \boldsymbol{u}_{r m s} \sim 0.14$. The greatest normalized departure from axisymmetry for the perturbed magnetic field is also at $M=4524$ for the 'D' cross section, $\tilde{\boldsymbol{B}}_{r m s}^{\prime} / \boldsymbol{B}_{r m s}^{\prime} \sim$ 0.015. In fact for the magnetic field, the fluctuations are of the same order of magnitude as the velocity field, but the magnitude of the perturbed magnetic field is larger, hence the normalized quantities are smaller. The distribution of the perturbations in the two- 


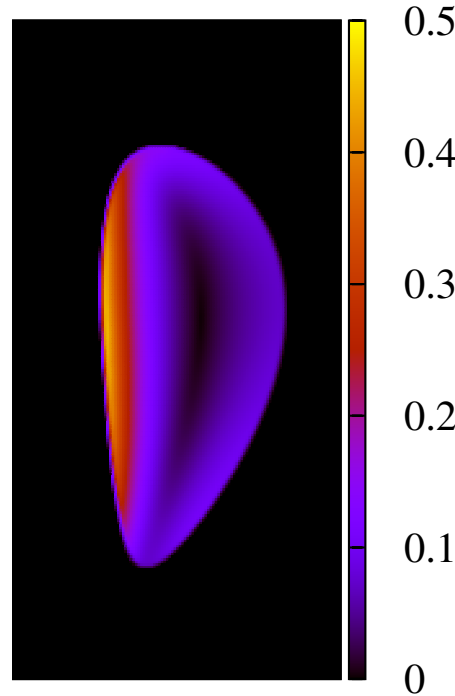

(a)

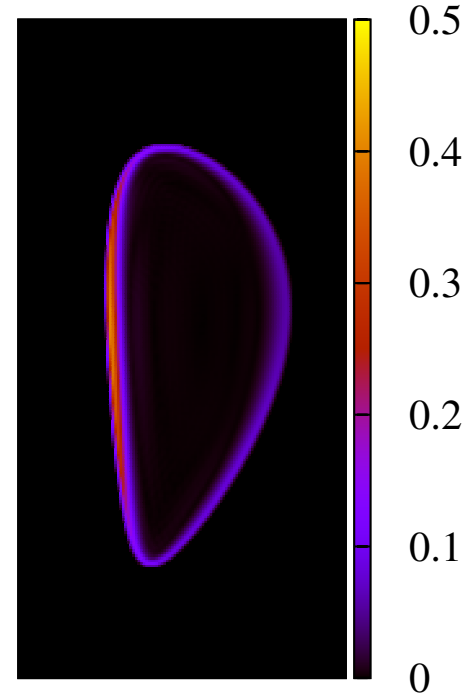

$(b)$

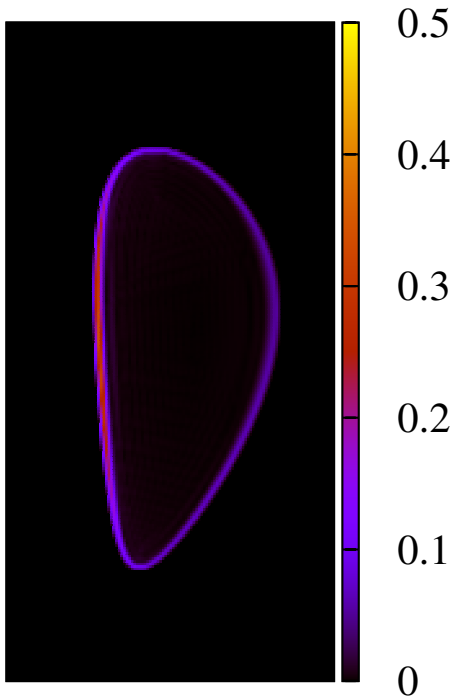

$(c)$

Figure 18. Azimuthally averaged Lorentz force vector norm for $M=23(a)$, $M=1131(b)$ and $M=4524(c)$.

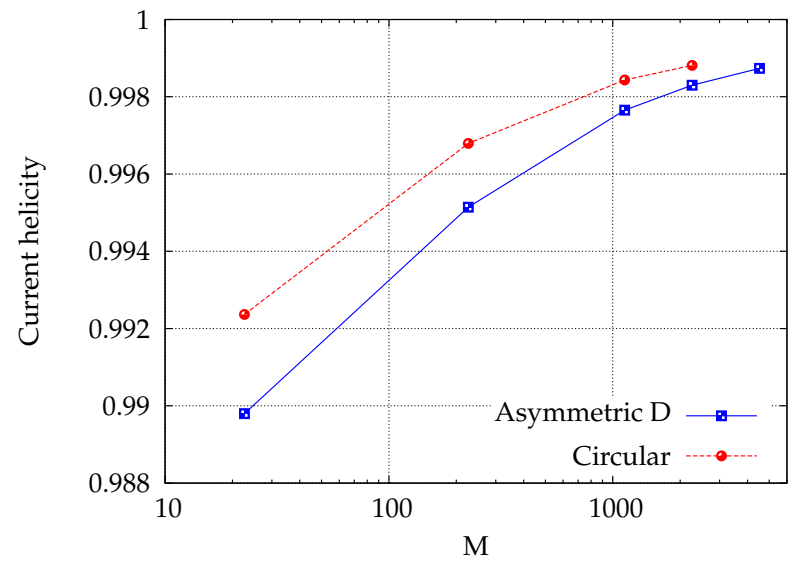

Figure 19. Current helicity as a function of $M$ for the asymmetric and symmetric cross sections.

dimensional plane (Figs. 23 and 24) show the velocity fluctuations mainly concentrated at the boundaries. These are the areas where the velocity is peaked (see e.g. velocity profiles Fig. $14(d))$ and where the velocity gradients are important. For the magnetic field the fluctuations are spread in a larger region, they are more important at the high and low field side of the torus.

\subsection{Influence of the safety factor on the dynamics}

The study of the influence of the safety factor $q$ is presented in this section where we consider only the asymmetric cross section geometry and the transport coefficients are 


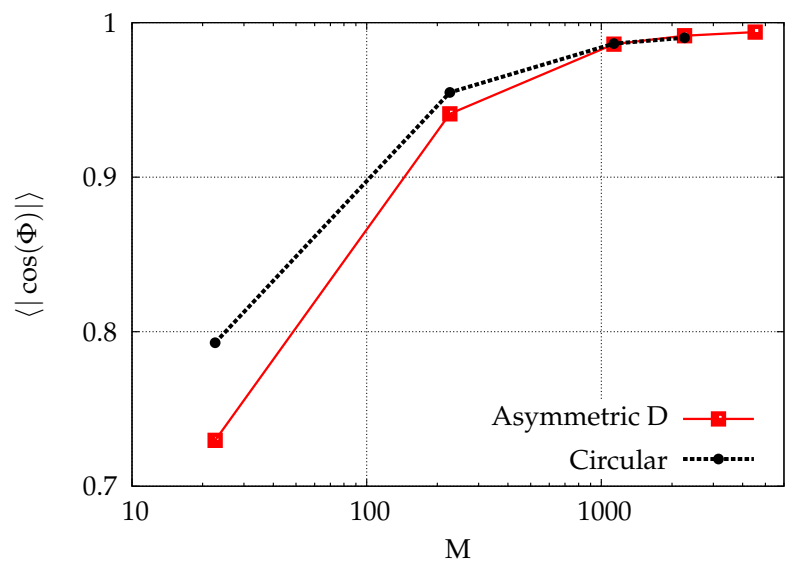

Figure 20. Volume-averaged absolute value of the cosine of the angle between the poloidal current density $\left(\boldsymbol{j}_{\text {pol }}\right)$ and the poloidal magnetic field $\left(\boldsymbol{B}_{p o l}\right)$.

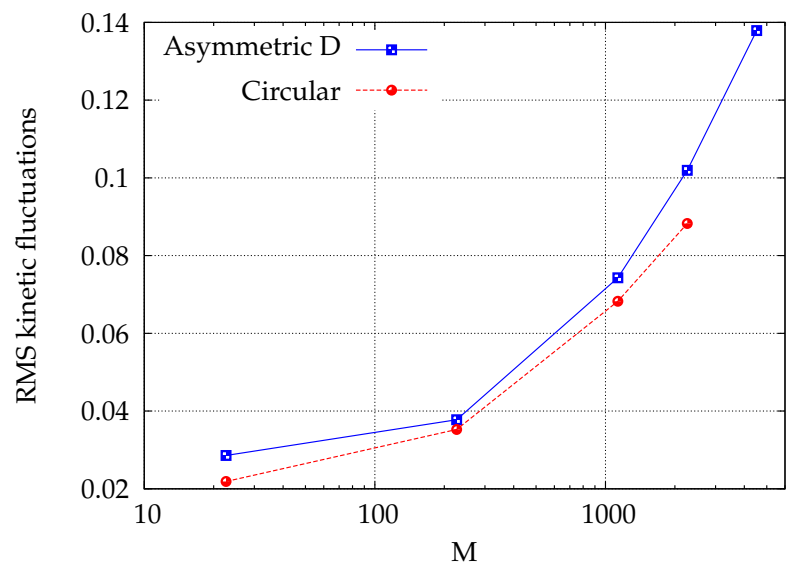

Figure 21. RMS value of the non azimuthally symmetric velocity fluctuations, normalized by the total root mean square velocity.

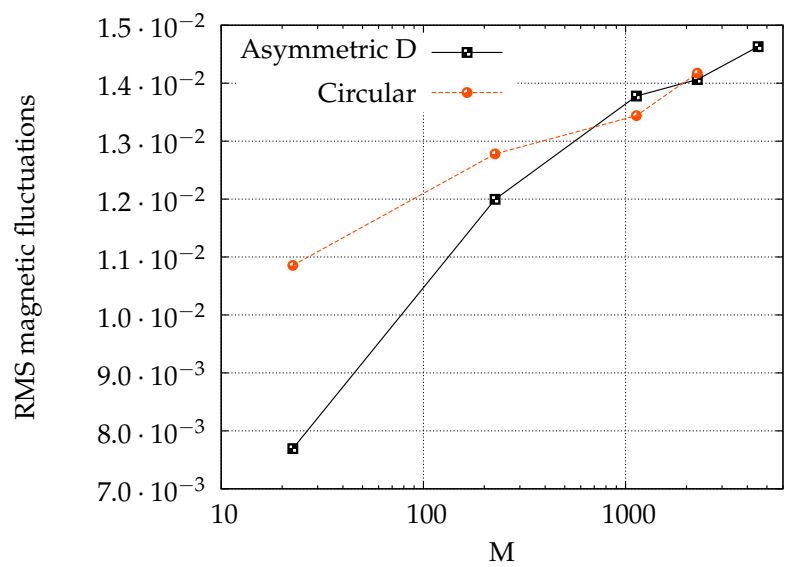

Figure 22. RMS value of the non azimuthally symmetric magnetic fluctuations, normalized by the total root mean square perturbed magnetic field.

kept constant $\left(\nu=\lambda=2 \cdot 10^{-3}\right)$. We recall that for all the simulations presented in this manuscript the magnetic Prandtl number is equal to one, $\operatorname{Pr}=1$. In this 


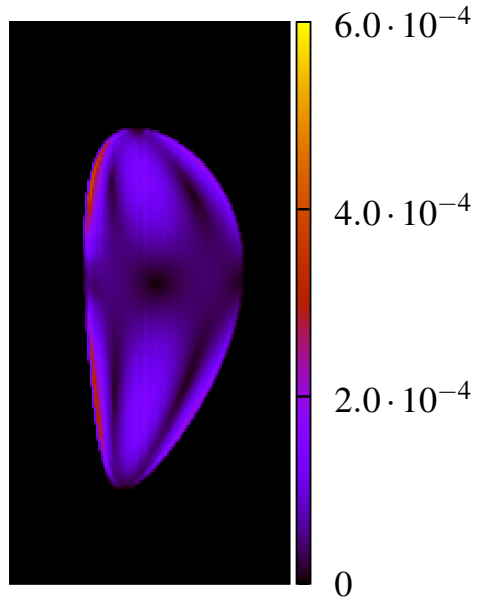

(a)

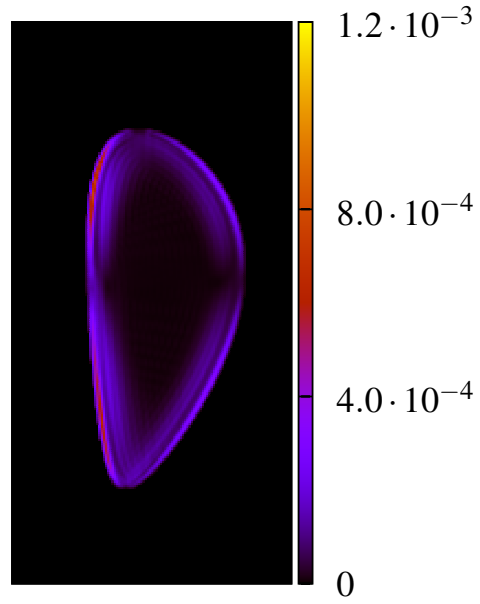

(b)

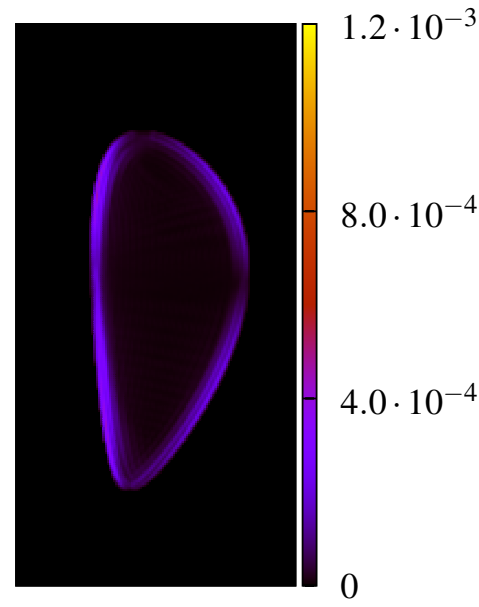

$(c)$

Figure 23. Azimuthally averaged square velocity fluctuations around the azimuthal mean value for $M=23(a), M=1131(b)$ and $M=4524(c)$.

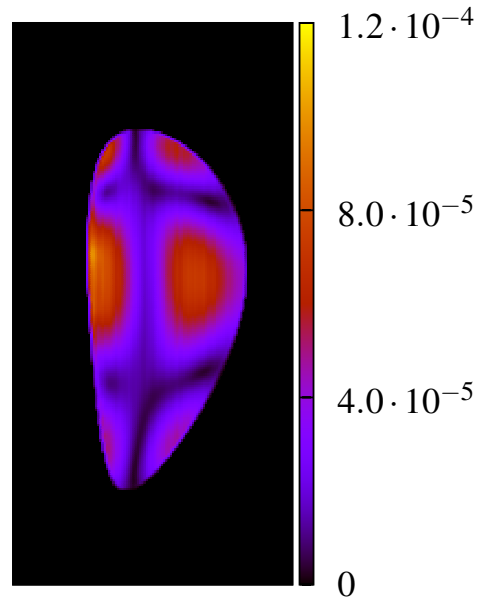

(a)

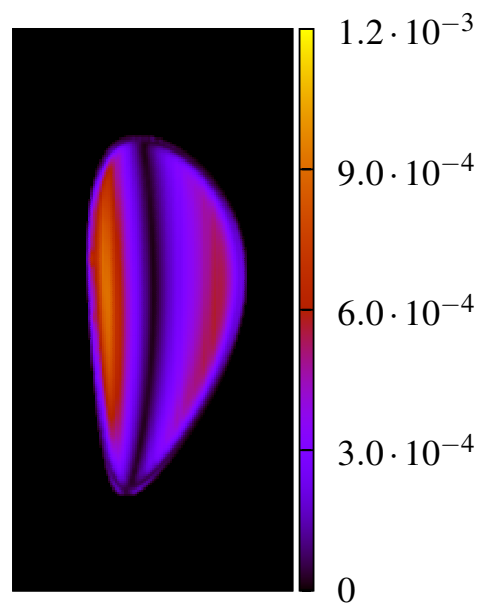

(b)

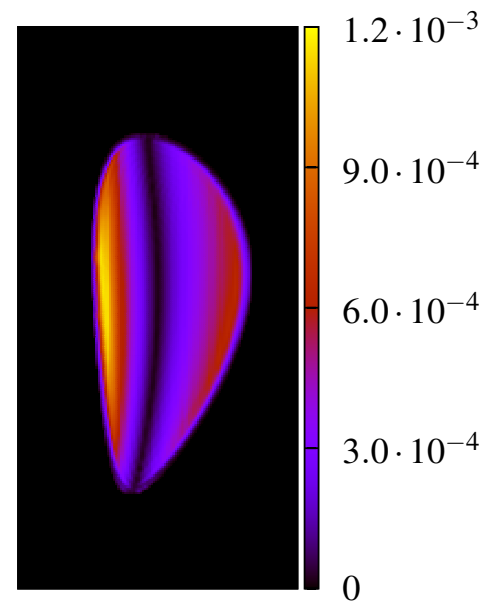

$(c)$

Figure 24. Azimuthally averaged square magnetic fluctuations around the azimuthal mean value for $M=23(a), M=1131(b)$ and $M=4524(c)$.

case the viscous Lundquist number varies because the reference magnetic field used for its calculation is the imposed toroidal component and to modify the safety factor the magnitude of this field is changed, as also done in experiments [22, 23]. The parameter $q$ takes four different values. We recall that the safety factor is defined as the ratio between the wall-averaged toroidal and poloidal imposed magnetic fields,

$$
q=\frac{\overline{\left.r B_{0_{T}}\right|_{\text {wall }}}}{R_{0} \overline{\left.B_{0_{P}}\right|_{\text {wall }}}} .
$$

The values of the viscous Lundquist number associated to each safety factor are presented in Tab. 1.

The evolution of the total kinetic energy and the magnetic energy of the perturbation is similar for all the studied cases (see Figs. 25 and 26). The main difference 
Table 1. Corresponding viscous Lundquist number for each safety factor value.

\begin{tabular}{r|cccc}
$q$ & 5.7 & 4.8 & 3.8 & 2.9 \\
\hline$M$ & 1131 & 942 & 754 & 565
\end{tabular}

is the magnitude of the energies that is higher if the safety factor is small. At the steady state the dependence of the kinetic energy on the safety factor is visualized in Fig. 27.
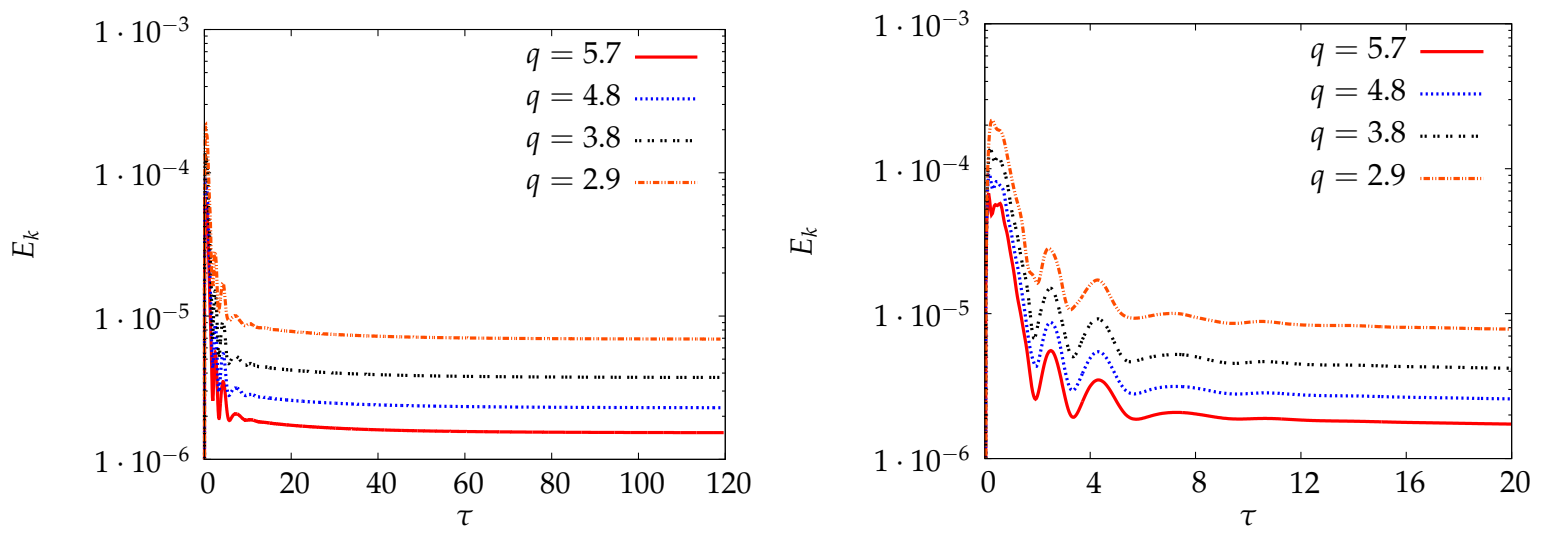

Figure 25. Left: kinetic energy evolution. Right: a zoom on the early time instants. Time is given in toroidal Alfvénic time units.
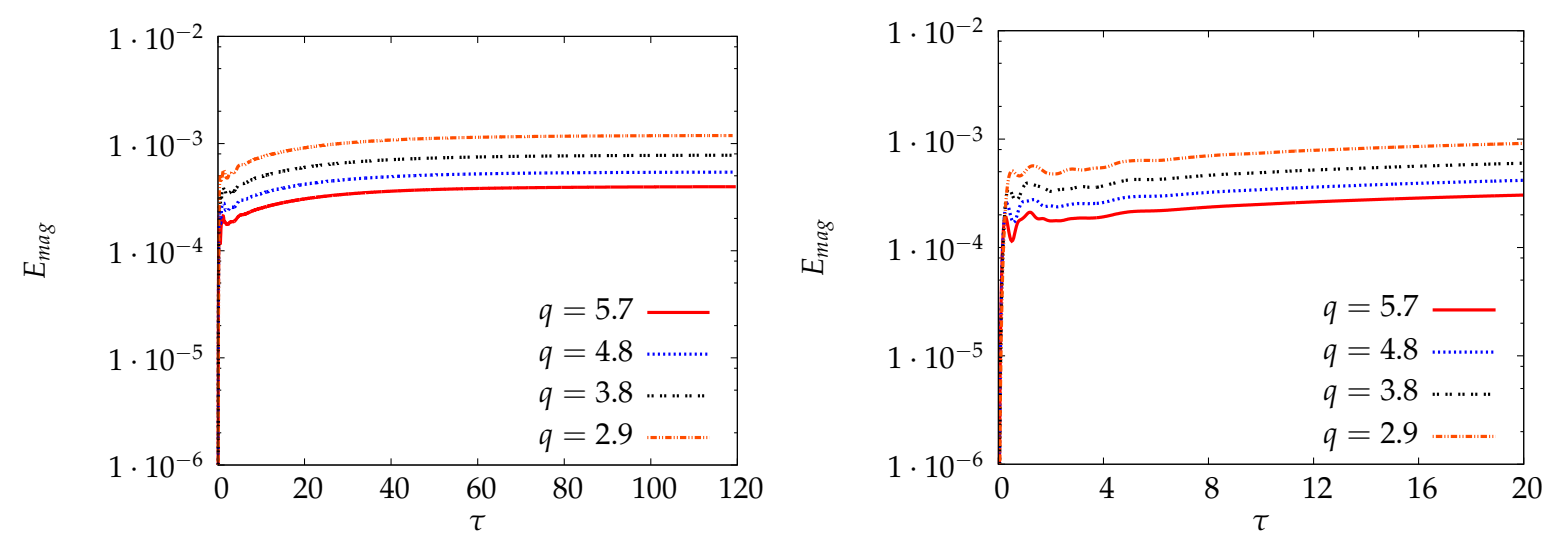

Figure 26. Left: perturbed magnetic energy evolution. Right: a zoom on the early time instants. Time is given in toroidal Alfvénic time units.

The growth of the kinetic energy with decreasing $q$ is in agreement with the reduction of the current helicity value (Fig. 28). Hence the Lorentz force term is stronger for a low safety factor. It is also observed that in the toroidal direction the Lorentz force increases, since the alignment between the poloidal current density and the poloidal magnetic field is less important for small $q$ (Fig. 29). This variation is smaller compared to the variation caused by the transport coefficients modification, as shown in Sec. 3.2.

As in the previous section the toroidal velocity dominates, but the ratio $\left\langle u_{T}^{2}\right\rangle /\left\langle\left|\boldsymbol{u}^{2}\right|\right\rangle$ decreases with decreasing $q$ (Fig. 30). Also the alignment between the magnetic and velocity field is less important (inset Fig. 30). 


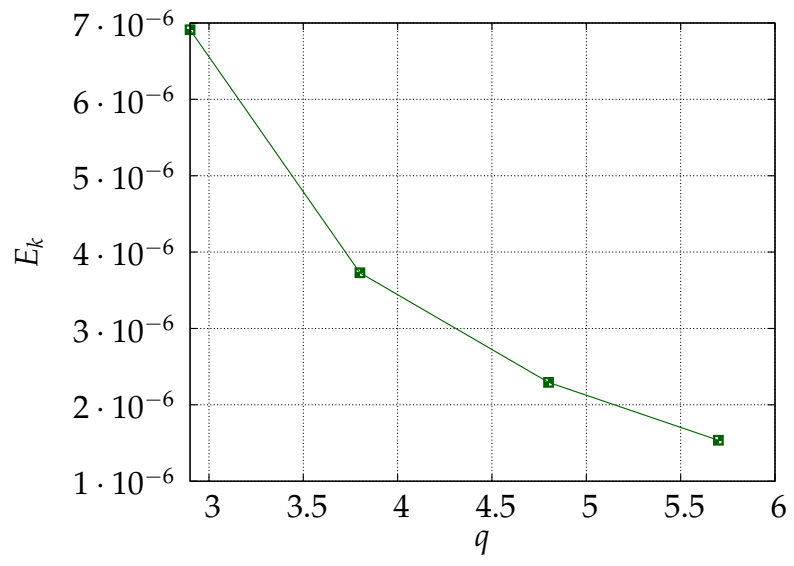

Figure 27. Kinetic energy as a function of $q$ for the asymmetric and symmetric cross sections.

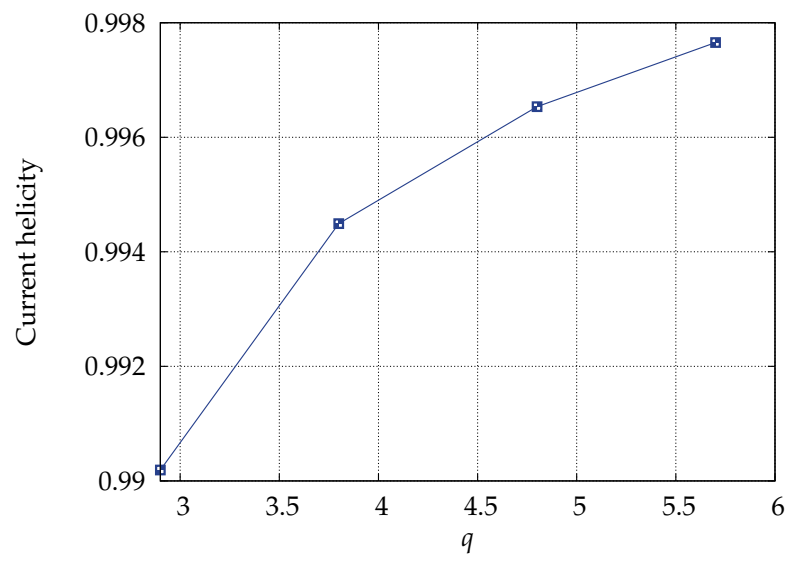

Figure 28. Current helicity as a function of the safety factor $q$.

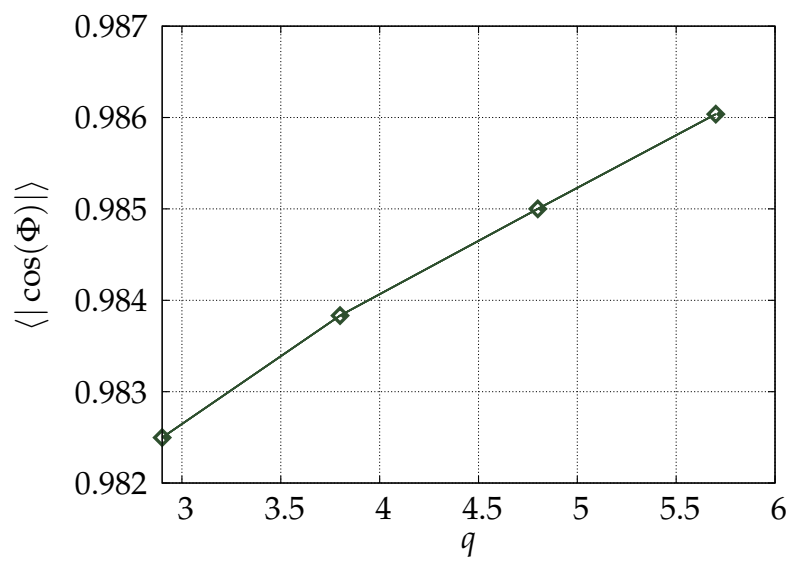

Figure 29. Volume-averaged absolute value of the cosine of the angle between the poloidal current density $\left(\boldsymbol{j}_{p o l}\right)$ and the poloidal magnetic field $\left(\boldsymbol{B}_{p o l}\right)$. 


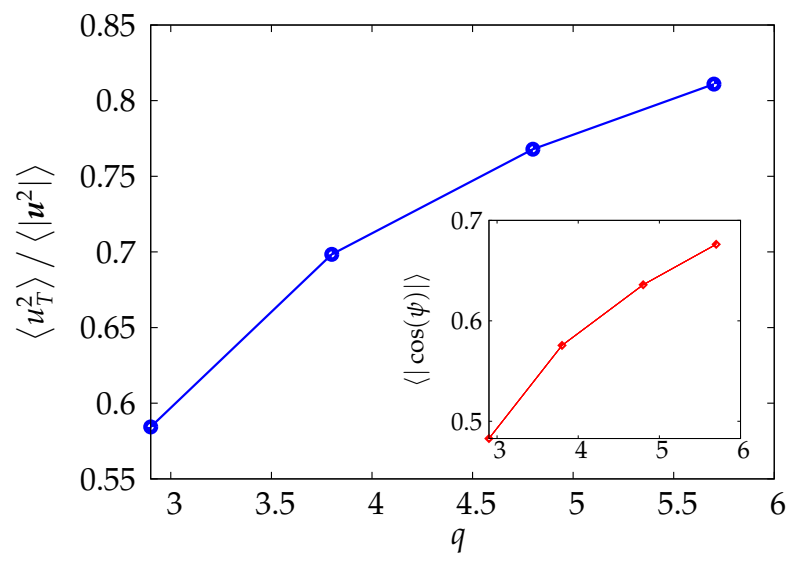

Figure 30. The ratio of the mean-square toroidal velocity to the total mean-square $\left\langle u_{T}^{2}\right\rangle /\left\langle\left|\boldsymbol{u}^{2}\right|\right\rangle$ as a function of $q$. In the inset we show the average over the domain of the absolute value of the cosine of the angle between the velocity field and magnetic field.

An important feature is the change of sign in the volume averaged toroidal angular momentum, found also in experimental observations [22, 23, 24], when the toroidal magnetic field, hence the safety factor, is varied (Fig. 31). In our case the averaged angular momentum changes completely in sign, it passes from negative to positive for increasing $q$. The two-dimensional azimuthally averaged toroidal velocities (Fig. 32) show the increase of the area in which the toroidal velocity is negative when the safety factor is decreased. For the lowest value of $q$ that we consider, the vertical cut (Fig. 32 $(d)$ ) shows larger velocities and a small downward shift of the position where the toroidal velocity changes sign. This displacement enlarges the negative velocity area. The growth of the negative toroidal velocity is better visualized in the cuts along the direction of the big radius (Fig. 33). For decreasing $q$ the velocities tend to be more peaked and near the center of the torus a region appears where the toroidal velocity is negative. We notice that the change of sign of the toroidal velocity mainly occurs in the center of the geometry. Close to the boundaries the toroidal component grows but does not reverse sign.

As presented in the previous section small fluctuations around the azimuthal average exist. We see in Fig. 34 that the magnitude of these fluctuations is relatively insensitive to the value of the safety factor. The change is just of a few percent for the normalized velocity fluctuations. It is larger for the normalized magnetic fluctuations, but it remains below $\sim 20 \%$ (Fig. 35). Hence the safety factor variation, in the considered range, does not increase substantially the non-axisymmetric perturbations.

\subsection{Influence of the reversal of the imposed toroidal magnetic field}

The simulation with inverted toroidal magnetic field is performed for $q=5.7$ and $M=1131$. The results show that the velocity reverses sign in the whole domain (Fig. 36). The counterrotating poloidal vortices are unchanged, only the toroidal 


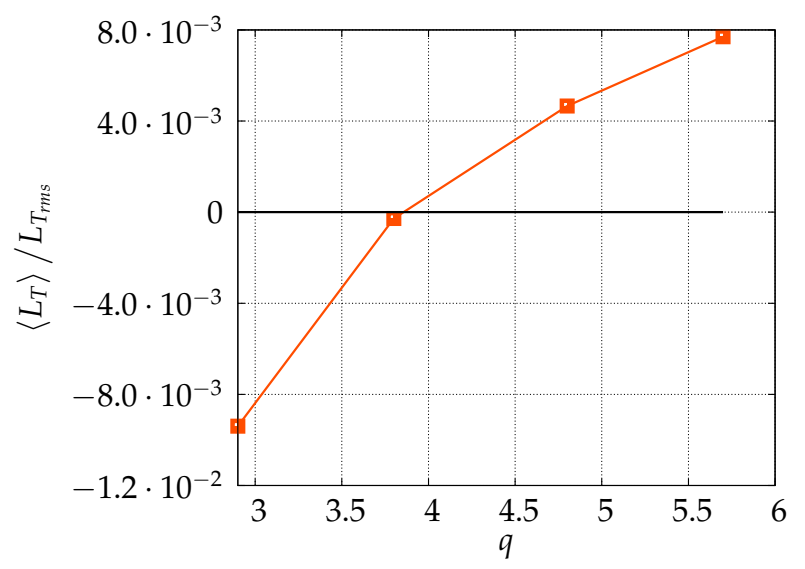

Figure 31. Normalized toroidal angular momentum $\left\langle L_{T}\right\rangle / L_{T_{r m s}}$ as a function of $q$.

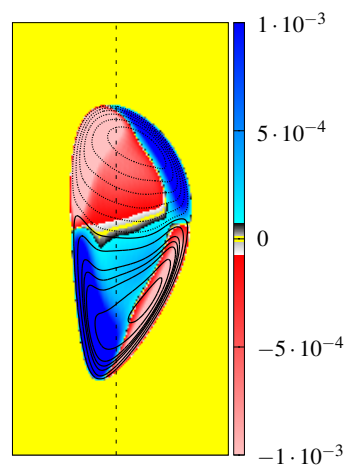

(a)

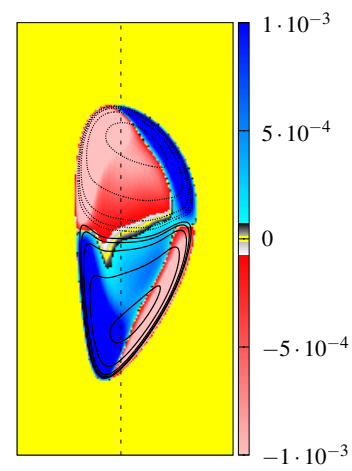

(b)

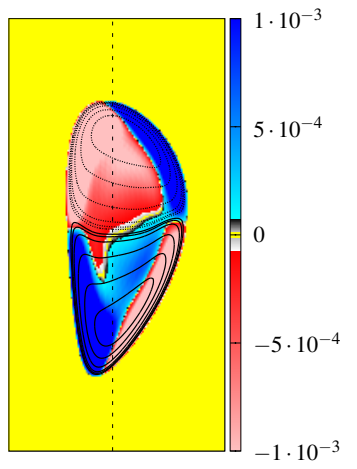

(c)

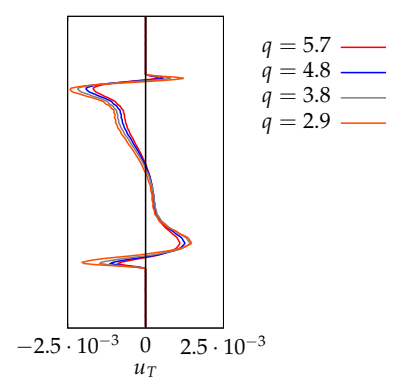

(d)

Figure 32. Azimuthally averaged flow visualizations: toroidal velocity $u_{T}$ and poloidal stream function contours (solid line positive, dotted line negative contours) for $q=5.7(a), q=3.8(b)$ and $q=2.9(c)$. (d) Toroidal velocity profiles along a vertical cut. The position of these cuts is indicated in $(a),(b)$ and $(c)$ by a dotted vertical line.

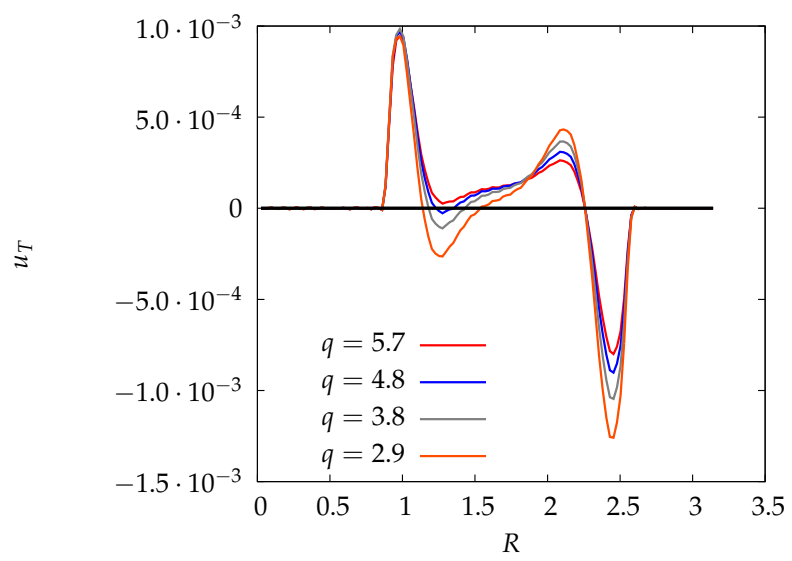

Figure 33. Toroidal velocity profiles along a horizontal cut, at the center of the domain. 


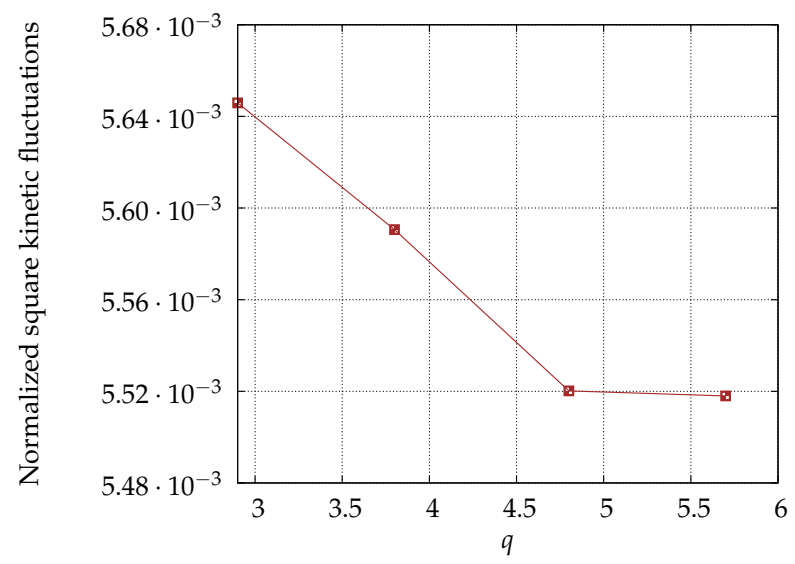

Figure 34. Square velocity fluctuations normalized by the total square velocity.

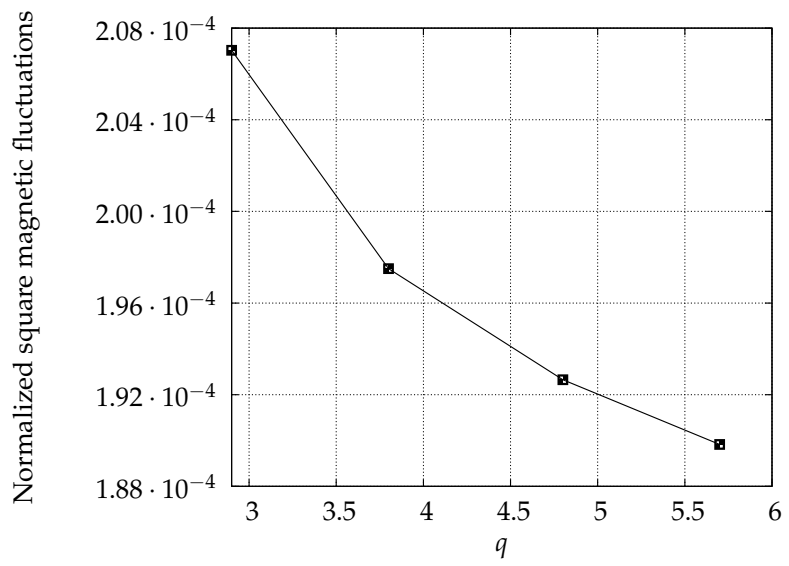

Figure 35. Square magnetic fluctuations normalized by the total square perturbed magnetic field.

velocities are affected. In Fig. $36(c)$ the profiles are exactly symmetric with respect to the vertical axis. Basically, what happens is that the perturbed toroidal magnetic field reverses its sign and this generates an inverse poloidal current density. The existing poloidal magnetic field associated with the inverted poloidal current density field gives an opposite toroidal Lorentz force. Finally, this Lorentz force will make the toroidal velocities reverse in all the domain. We can write the three components of the Lorentz force in cylindrical coordinates:

$$
\left\{\begin{array}{l}
F_{R}=j_{T} B_{Z}-\frac{1}{R} \frac{\partial\left(R B_{T}\right)}{\partial R} B_{T} \\
F_{T}=\frac{\partial B_{T}}{\partial Z} B_{Z}+\frac{1}{R} \frac{\partial\left(R B_{T}\right)}{\partial R} B_{R} \\
F_{Z}=-\frac{\partial B_{T}}{\partial Z} B_{T}+j_{T} B_{R} .
\end{array}\right.
$$

The inversion of the sign of $B_{T}$ transforms the original Lorentz force vector $\left(F_{R}, F_{T}, F_{Z}\right)$ into $\left(F_{R},-F_{T}, F_{Z}\right)$. Only the toroidal component is affected. Hence the poloidal velocities are unchanged but the toroidal velocities are inverted. 


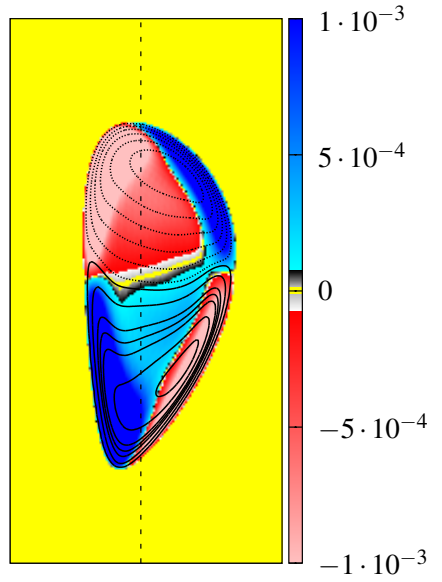

(a)

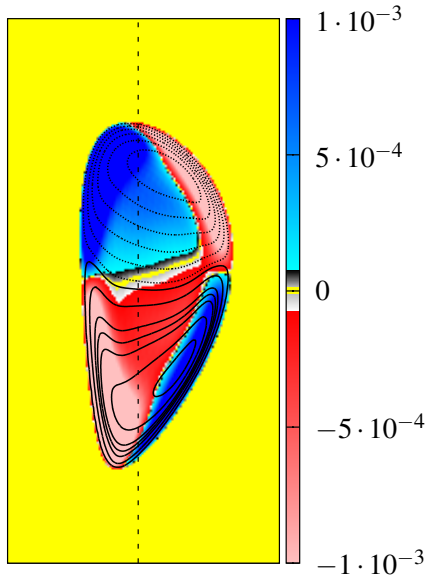

(b)

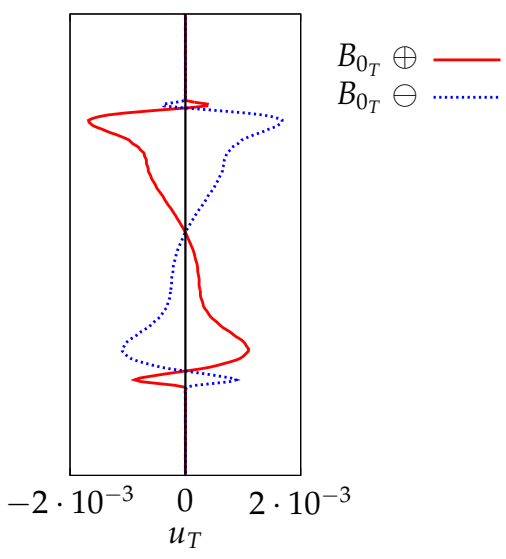

(c)

Figure 36. Azimuthally averaged flow visualizations: toroidal velocity $u_{T}$ and poloidal stream function contours (solid line positive, dotted line negative contours) for imposed positive $B_{0_{T}}(a)$ and negative $B_{0_{T}}(b)$. (c) Toroidal velocity profiles along a vertical cut. The position of these cuts is indicated in $(a)$ and $(b)$ by a dotted vertical line.

\section{Conclusion}

In the present paper it was demonstrated numerically, solving the fully nonlinear timedependent resistive MHD equations, that in a toroidal geometry, assuming constant transport coefficients, if the imposed toroidal magnetic and toroidal electric fields are irrotational, the conducting flow inside a torus necessarily moves. The reason for this is that the curl of the Lorentz force resulting of the imposed fields is nonzero. It follows that the gradient of a scalar (in this case the pressure) can not balance the equation. Consequently vorticity is created. This vorticity in the toroidal direction creates poloidal velocities. The poloidal velocities interact with the imposed toroidal magnetic field creating a perturbation that gives rise to a poloidal current density. This current density associated with the existing poloidal magnetic field produces a toroidal Lorentz force. As a consequence toroidal velocities appear. The angle between the poloidal current density and poloidal magnetic field plays an important role in the determination of the toroidal velocity direction.

For a low viscous Lundquist number the system tends to produce small toroidal velocities, the dominant flow being a pair of counterrotating vortices in the poloidal plane. A dramatic change occurs when the viscous Lundquist number is increased. There is a transition from a dominantly poloidal to dominantly toroidal flow. This transition is in agreement with the tendency of the velocity field to align with the magnetic field.

Two different toroidal geometries are considered in the present study, one with an up-down symmetric and the other with an asymmetric cross section. A fundamental difference exists between both studied cases: the volume-averaged angular momentum is zero for the symmetric case, while for the asymmetric cross section a finite volume- 
averaged angular momentum appears. There is a breaking in the up-down symmetry of the flow and a toroidal preferred direction emerges. This volume-averaged normalized angular momentum tends to increase with the viscous Lundquist number.

Nevertheless the kinetic energy decreases with increasing nonlinearity, since the total magnetic and current density fields tend to align in the center of the domain. The limitation in the numerical resources prevents the study of this system for larger viscous Lundquist numbers. It remains an open question if there will be a continuous increase of the alignment between the magnetic and current density field or if a transition exists.

When the safety factor is decreased while maintaining the transport coefficients constant, the kinetic and fluctuating magnetic energy become higher. The main qualitative effect is the influence on the toroidal velocity direction. There is a change in the volume-averaged angular momentum that reverses sign. For low $q$ it is negative and at large safety factor it becomes positive. Mainly in the center of the domain, for decreasing $q$, the region in which the velocity is negative becomes larger, at expense of the region with positive toroidal velocity. Near the boundaries the toroidal velocity direction remains unchanged.

The last part of the study was dedicated to the influence of the reversal of the toroidal magnetic field. It is shown that it plays a role only in the toroidal velocities. The reversal changes the sign of the poloidal current density, that gives rise to the toroidal Lorentz force. In consequence the toroidal force reverses in the whole volume making the toroidal velocities reverse their direction compared to the original case.

\section{Acknowledgements}

We acknowledge financial support from the French Research Agency (ANR), project SiCoMHD, contract ANR-11-BLAN-045, as well as IDRIS for computing time.

\section{Appendix A. Calculation of the imposed poloidal magnetic field}

We construct the magnetic field satisfying the following properties: $(i)$ it corresponds to a current density profile $\propto 1 / R,($ ii $)$ it is parallel to the wall and ( $i i i)$ it is solenoidal. With respect to our previous investigation [25] the magnetic topology is changed. In fact in the previous paper the imposed poloidal magnetic field satisfied the imposed toroidal current density profile $\boldsymbol{J}_{0_{T}}$ and the solenoidal constraint but the normal component did not vanish (as is shown in Fig. A1 $(c)$ ). To solve this problem and to satisfy the three desired conditions we obtain $\boldsymbol{B}_{0_{p o l}}$ from the current density by writing in terms of a vector potential $\boldsymbol{B}_{0_{p o l}}=\nabla \times\left.\boldsymbol{A}_{0}\right|_{\text {pol }}$, where $\boldsymbol{A}_{0}=A_{0_{T}} \boldsymbol{e}_{T}$.

The poloidal magnetic field is calculated from the imposed toroidal current density distribution $\boldsymbol{J}_{0_{T}}$. It can be obtained using the vector potential, $\boldsymbol{B}_{0_{\text {pol }}}=\nabla \times\left.\boldsymbol{A}_{0}\right|_{\text {pol }}$, where $\boldsymbol{A}_{0}=A_{0_{T}} \boldsymbol{e}_{T}$. Using the Coulomb Gauge we have the following Poisson equation $[26]$,

$$
\nabla^{2}\left(A_{0_{T}} \boldsymbol{e}_{T}\right)=-\boldsymbol{J}_{0_{T}}
$$




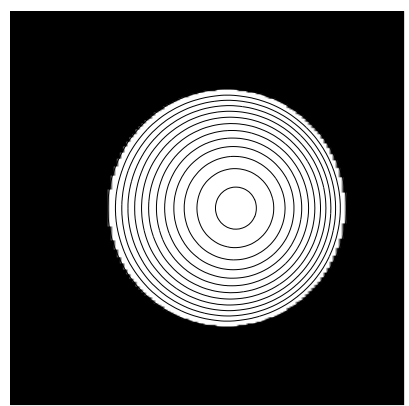

(a)

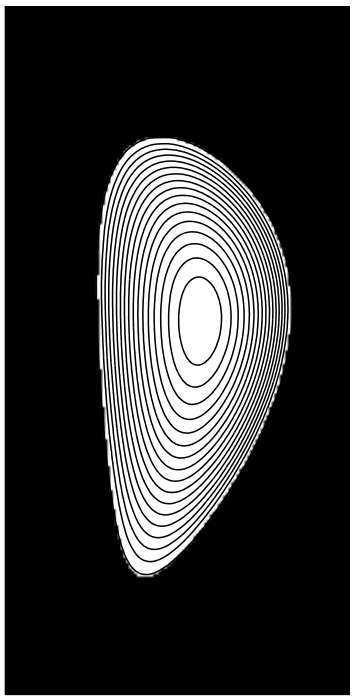

(b)

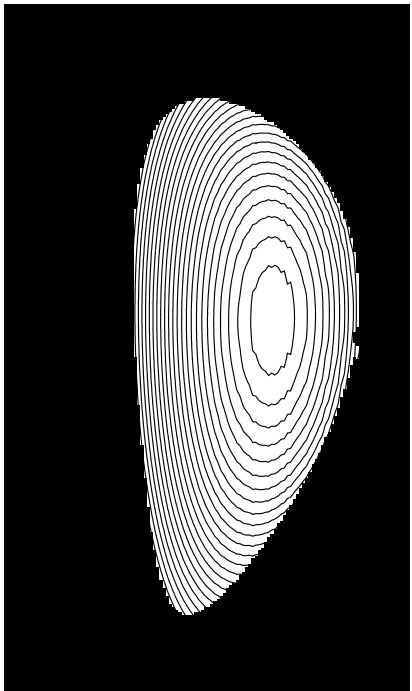

$(c)$

Figure A1. Poloidal magnetic field lines $\left(\chi=R A_{0_{T}}=\right.$ constant $)$ for the different cross sections: $(a)$ symmetric, $(b)$ asymmetric. In $(c)$ we show the field lines for the asymmetric geometry used in Ref.[25].

The associated boundary condition is the normal component of the magnetic field vanishing at the boundary of the torus.

It is equivalent and more convenient to work with the magnetic flux function $\chi(R, Z)=R A_{0_{T}}$, directly. The axisymmetric poloidal magnetic field is easily derived from the flux function $\chi(R, Z)$,

$$
\boldsymbol{B}_{0_{p o l}}=\nabla \chi \times \nabla T
$$

with $\nabla T=(1 / R) \boldsymbol{e}_{T}$. Substituting this into Ampère's law, $\nabla \times \boldsymbol{B}_{0_{p o l}}=J_{0_{T}} \boldsymbol{e}_{T}$ yields

$$
\Delta^{*} \chi=\frac{\partial}{\partial R}\left(\frac{1}{R} \frac{\partial \chi}{\partial R}\right)+\frac{1}{R} \frac{\partial^{2} \chi}{\partial Z^{2}}=-J_{0_{T}} .
$$

The boundary condition $\left.\boldsymbol{B}_{0_{\text {pol }}} \cdot \boldsymbol{n}\right|_{\text {wall }}=0$ implies a Dirichlet boundary condition on the magnetic flux function $\left.\chi\right|_{\text {wall }}=$ constant .

Numerically the calculation of the poloidal magnetic field $\boldsymbol{B}_{0_{p o l}}$ is performed solving the previous Poisson equation for the magnetic flux function $\chi$. This equation is solved with a Fourier spectral method and the volume-penalization technique is used to impose the Dirichlet boundary condition at the wall [7]. The resulting computed equation is the following,

$$
\frac{\partial \chi}{\partial t}=\lambda \Delta^{*} \chi+\lambda J_{0_{T}}-\underbrace{\frac{\Xi(\boldsymbol{x})}{\eta}\left(\chi-\chi_{\text {wall }}\right)}_{\text {Penalisation term }} .
$$

Here $\lambda$ is a diffusion coefficient $(\lambda=1), \Xi$ is the mask function (it takes the value one in the region where the Poisson equation needs to be solved and zero in the rest of the computational domain) and $\eta$ is the penalization parameter $\left(\eta=5 \cdot 10^{-4}\right)$. The size of 
the domain is $(2 \pi)^{3}$ for the asymmetric and $(2 \pi \times 2 \pi \times \pi)$ for the symmetric cross section with a resolution of $256^{3}$. The value of the Dirichlet boundary condition is $\chi_{\text {wall }}$. This

equation is evolved in time, reaching a steady state, numerically $\left\|\chi^{n+1}-\chi^{n}\right\|<10^{-6}$. We then recover with sufficient accuracy the solution of the Poisson equation (A.3) taking into account the Dirichlet boundary condition via the penalization term. The solution of this pre-computation will give our basis magnetic field $\boldsymbol{B}_{0}$ which will be kept constant during the actual simulation.

The resulting poloidal magnetic topology is presented in Fig. A1 $((a)$ and $(b))$ respectively for the considered symmetric and asymmetric geometries (Fig. 1).

\section{References}

[1] J.E. Rice, A. Ince-Cushman, L.-G. Eriksson, Y. Sakamoto, A. Scarabosio, A. Bortolon, K.H. Burrell, B.P. Duval, C. Fenzi-Bonizec, M.J. Greenwald, et al. Inter-machine comparison of intrinsic toroidal rotation in tokamaks. Nuclear Fusion, 47(11):1618, 2007.

[2] D. Pfirsch and A. Schlüter. Der Einfluss der elektrischen Leitfähigkeit auf das Gleichgewichtsverhalten von Plasmen niedrigen Drucks in Stelleratoren. Technical Report MPI/PA/7/62, MaxPlanck-Institut, Munich, 1962.

[3] P. Angot, C.-H. Bruneau, and P. Fabrie. A penalization method to take into account obstacles in incompressible viscous flows. Numerische Mathematik, 81(4):497-520, 1999.

[4] S. Neffaa, W.J.T. Bos, and K. Schneider. The decay of magnetohydrodynamic turbulence in a confined domain. Phys. of Plasmas, 15:092304, 2008.

[5] K. Schneider. Numerical simulation of the transient flow behaviour in chemical reactors using a penalisation method. Computers \& Fluids, 34(10):1223-1238, 2005.

[6] W.J.T. Bos, S. Neffaa, and K. Schneider. Rapid generation of angular momentum in bounded magnetized plasma. Phys. Rev. Lett., 101:235003, 2008.

[7] J.A. Morales, M. Leroy, W.J.T. Bos, and K. Schneider. Simulation of confined magnetohydrodynamic flows using a pseudo-spectral method with volume penalization. http://hal.archivesouvertes.fr/hal-00719737, V1, 2012.

[8] J. Berkowitz, H. Grad, and H. Rubin. Proceedings of the second united nations international conference on the peaceful uses of atomic energy, Geneva. 1958.

[9] V.D. Shafranov. Plasma equilibrium in a magnetic field. Reviews of Plasma Phys., 2:103, 1966.

[10] J.W. Bates and D.C. Montgomery. Toroidal visco-resistive magnetohydrodynamic steady states contain vortices. Phys. of Plasmas, 5:2649, 1998.

[11] L.P.J. Kamp and D.C. Montgomery. Toroidal steady states in visco-resistive magnetohydrodynamics. Journal of Plasma Phys., 70(2):113-142, 2004.

[12] J. Manickam. Stability of $\mathrm{n}=1$ internal modes in tokamaks. Nuclear Fusion, 24(5):595, 1984.

[13] S. Cappello and D.F. Escande. Bifurcation in viscoresistive MHD: The Hartmann number and the Reversed Field Pinch. Phys. Rev. Lett., 85:3838-3841, 2000.

[14] X. Shan and D. Montgomery. On the role of the hartmann number in magnetohydrodynamic activity. Plasma Phys. and Controlled Fusion, 35(5):619, 1993.

[15] D.C. Montgomery and X. Shan. Toroidal resistive MHD equilibria. Comments Plasma Phys. Control Fusion, 15:315, 1994.

[16] J.W. Bates and R.H. Lewis. A toroidal boundary-value problem in resistive magnetohydrodynamics. Phys. of Plasmas, 3:2395, 1996.

[17] D.C. Montgomery, J.W. Bates, and R.H. Lewis. Resistive magnetohydrodynamic equilibria in a torus. Phys. of Plasmas, 4(4):1080, 1997.

[18] L.P.J. Kamp and D.C. Montgomery. Toroidal flows in resistive magnetohydrodynamic steady states. Phys. of Plasmas, 10:157, 2003. 
[19] Y. Camenen, A.G. Peeters, C. Angioni, F.J. Casson, W.A. Hornsby, A.P. Snodin, and D. Strintzi. Transport of parallel momentum induced by current-symmetry breaking in toroidal plasmas. Phys. Rev. Lett., 102:125001, 2009.

[20] Y. Camenen, A. Bortolon, B.P. Duval, L. Federspiel, A.G. Peeters, F.J. Casson, W.A. Hornsby, A.N. Karpushov, F. Piras, O. Sauter, et al. Experimental demonstration of an up-down asymmetry effect on intrinsic rotation in the TCV tokamak. Plasma Phys. and Controlled Fusion, 52(12):124037, 2010.

[21] J.P. Dahlburg, D. Montgomery, G.D. Doolen, and L. Turner. Driven, steady-state RFP computations. Journal of Plasma Phys., 40(01):39-68, 1988.

[22] J.E. Rice, B.P. Duval, M.L. Reinke, Y.A. Podpaly, A. Bortolon, R.M. Churchill, I. Cziegler, P.H. Diamond, A. Dominguez, P.C. Ennever, et al. Observations of core toroidal rotation reversals in Alcator C-mod ohmic L-mode plasmas. Nuclear Fusion, 51(8):083005, 2011.

[23] J.E. Rice, M.J. Greenwald, Y.A. Podpaly, M.L. Reinke, P.H. Diamond, J.W. Hughes, N.T. Howard, Y. Ma, I. Cziegler, B.P. Duval, et al. Ohmic energy confinement saturation and core toroidal rotation reversal in Alcator C-mod plasmas. Phys. of Plasmas, 19:056106, 2012.

[24] A. Scarabosio, A. Bortolon, B.P. Duval, A. Karpushov, and A. Pochelon. Toroidal plasma rotation in the TCV tokamak. Plasma Phys. and Controlled Fusion, 48(5):663, 2006.

[25] J.A. Morales, W.J.T. Bos, K. Schneider, and D.C. Montgomery. Intrinsic rotation of toroidally confined magnetohydrodynamics. Phys. Rev. Lett., 109:175002, 2012.

[26] D.C. Montgomery, J.W. Bates, and S. Li. Toroidal vortices in resistive magnetohydrodynamic equilibria. Phys. of Plasmas, 9(4):1188, 1996. 